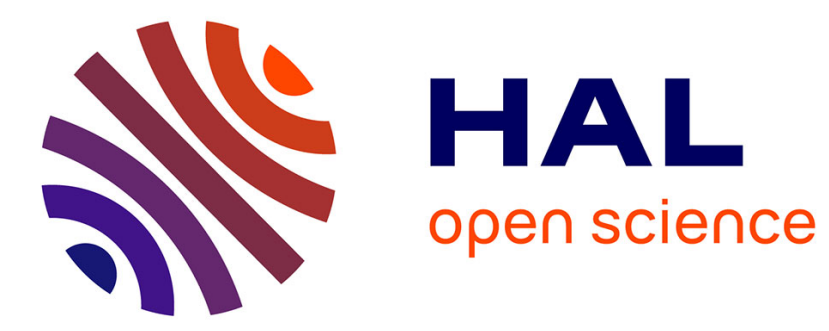

\title{
Semi-group stability of finite difference schemes in corner domains
}

Antoine Benoit

\section{To cite this version:}

Antoine Benoit. Semi-group stability of finite difference schemes in corner domains. Numerical mathematics : a journal of Chinese universities, 2018, 11 (3), pp.618-654. 10.4208/nmtma.2017-OA-0072 . hal-01417105

\section{HAL Id: hal-01417105 \\ https://hal.science/hal-01417105}

Submitted on 15 Dec 2016

HAL is a multi-disciplinary open access archive for the deposit and dissemination of scientific research documents, whether they are published or not. The documents may come from teaching and research institutions in France or abroad, or from public or private research centers.
L'archive ouverte pluridisciplinaire HAL, est destinée au dépôt et à la diffusion de documents scientifiques de niveau recherche, publiés ou non, émanant des établissements d'enseignement et de recherche français ou étrangers, des laboratoires publics ou privés. 


\title{
Semi-group stability of finite difference schemes in corner domains
}

\author{
Antoine BENOIT \\ Université Libre de Bruxelles, Département de Mathématiques (ULB) \\ boulevard du Triomphe, 1050 Brussels, Belgium \\ $\&$ \\ Project-team MEPHYSTO, Inria Lille - Nord Europe \\ 40, avenue Halley, 59650 Villeneuve d'Ascq, France \\ Email: antoine.benoit@inria.fr \\ Phone: +322650.58 .52$
}

December 5, 2016

\begin{abstract}
In this article we are interested in the semi-group stability for finite difference schemes approximations of hyperbolic systems of equations in corner domains. We give generalizations of the results of [CG11] and Cou15] from the half space geometry to the quarter space geometry. The most interesting fact is that the proofs of CG11 and Cou15 can be adaptated with minor changes to apply in the quarter space geometry. This is due to the fact that both methods in [CG11] and Cou15] are based on energy methods and the construction of auxiliary problems with strictly dissipative boundary conditions which are known to be suitable for the strong well-posed for initial boundary value problems in the quarter space.
\end{abstract}

Aknowledgement The author acknowledges financial support from the European Research Council under the European Community's Seventh Framework Programme (FP7/2014-2019 Grant Agreement QUANTHOM 335410). AMS subject classification :65M06-65M12

\section{Contents}

$\mathbf{1}$ Introduction $\mathbf{2}$

$1.1 \quad$ Some results about strong well-posed and stability for the half space geometry . . . . . . . . 3

1.2 Generalization of semi-group stability results to corner domains . . . . . . . . . . . . . . . . 5

1.3 Organization of the article . . . . . . . . . . . . . . . . . . . . 6

2 Finite difference schemes and corner

2.1 General notations and definitions . . . . . . . . . . . . . . . . . . . . . . . . 6

2.2 Boundary and corner conditions . . . . . . . . . . . . . . . . . . . . . . . 8

3 Main results 99

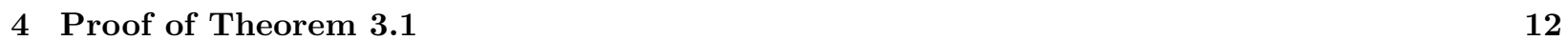

$4.1 \quad$ Finite difference schemes with discrete Dirichlet boundary and corner conditions . . . . . . . 12

4.1 .1 Homogeneous schemes in the interior . . . . . . . . . . . . . . . . . . . 12

4.1 .2 Homogeneous Dirichlet conditions . . . . . . . . . . . . . . . . . . . . . . 15

4.2 Reinforcement of traces estimates . . . . . . . . . . . . . . . . . . . . . 16

4.3 End of the proof by error estimate . . . . . . . . . . . . . . . . . . . . . . . . 18 
5.1 .1 Proof of Theorem 3.2 for homogeneous initial conditions . . . . . . . . . . . . . . 21

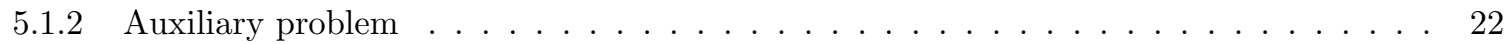

5.2 End of the proof by error estimate . . . . . . . . . . . . . . . . . . 26

\section{Introduction}

In this article we are interested in finite difference schemes approximation for linear hyperbolic problems in the quarter space. Such problems read:

$$
\left\{\begin{array}{l}
L(\partial) u:=\partial_{t} u+A_{1} \partial_{1} u+A_{2} \partial_{2} u+\sum_{j=3}^{d} A_{j} \partial_{j} u=f, \text { in }\left[0, \infty\left[\times \Omega \times \mathbb{R}^{d-2}\right.\right. \\
B_{1} u_{\mid x_{1}=0}=g_{1}, \text { on }\left[0, \infty\left[\times \partial \Omega_{1} \times \mathbb{R}^{d-2}\right.\right. \\
B_{2} u_{\mid x_{2}=0}=g_{2}, \text { on }\left[0, \infty\left[\times \partial \Omega_{2} \times \mathbb{R}^{d-2}\right.\right. \\
u_{\mid t=0}=u_{0}, \text { on } \Omega \times \mathbb{R}^{d-2}
\end{array}\right.
$$

where $\Omega$ denotes the quarter space $\mathbb{R}_{+}^{2}$ and $\partial \Omega_{1}$ (resp. $\partial \Omega_{2}$ ) is the component of the boundary associated to $\left\{x_{1}=0\right\}$ (resp. $\left\{x_{2}=0\right\}$ ). In $(1)$ the coefficients in the interior, the $A_{j}$ are matrices in $\mathbf{M}_{n \times n}(\mathbb{R})$ while the coefficient on the boundary $B_{1}\left(\operatorname{resp} . B_{2}\right)$ is an element of $\mathbf{M}_{p_{1} \times n}(\mathbb{R})\left(\operatorname{resp} . \mathbf{M}_{p_{2} \times 2}(\mathbb{R})\right)$ where $p_{1}($ resp. $p_{2}$ ) denotes the number of strictly positive eigenvalues of $A_{1}$ (resp. $A_{2}$ ).

Finite difference schemes approximations in the quarter space are thus just discretizations of (1) and have pratical motivations in scientific computations. Indeed, due to the impossibility to modeling the full space $\mathbb{R}^{d}$ during a numerical simulation, all the schemes implemented in a computer lie in a large rectangle and thus numerically boundary conditions have to be specified even for the numerical approximation of a Cauchy problem. Thus the theoretical study of such schemes set in a domain with corners also have more pratical views. About these pratical views we can be more specific and describe, for example the question of absorbing boundary conditions for wave propagation (see for example [EM77]- Hig86] and [Ehr10]). These conditions are non physical ones and aim to minimize, as much as possible, the "parasite" reflections which occur when the wave hits the artificial boundaries implemented in the simulation of the Cauchy problem. Consequently these conditions are choosen in such a way that the reflections against the boundaries modify or influence as little as possible the approximation in the interior of the box. A similar method is the study of perfectly matched layer (see for example Ber94]) which are boundary conditions which will only modify the approximation in a small neighborhood of the boundary.

In this article we are interested in the stability of difference schemes approximation set in a space with corner. But before to turn to a more precise description of the notion of stability for schemes with corner let us recall some elements of comparison with the notion of strong well-posedness for continous problems.

Strong well-posedness means existence and uniqueness of the solution of (1) and that this solution is as regular (in the $L^{2}$-norm) as the datas of the problem. Such a control of the solution by the data is refered as an energy estimate for (1). In the author knowledge, even for homogeneous initial conditions (that is to say $\left.u_{0} \equiv 0\right)$ the strong well-posedness of (1), under suitable conditions, has not been established yet. The main contribution about this question is due to Osh73, in which the author obtains, thanks to the introduction of a new inversibility condition (we refer to [Osh73] or to [[Ben15], Chapitre 5] for more details), an energy estimate for the $L^{2}$-norm of the solution. However the regularity of the source terms of (1) asked to control the $L^{2}$-norm of the solution is not explicit. As a consequence, there is a non explicit number of losses of derivatives in the energy estimate and we can not conclude to the strong well-posedness.

However in a particular framework, more precisely for strictly dissipative boundary conditions, that is to say boundary conditions which make the energy decrease, the strong well-posedness (with homogeneous 
initial datas) is established see [ $[$ Ben15], Chapitres 4 and 5]. We also refer to [HR] for a result dealing with three dimensional corners in which, thanks to the strict dissipativity and under an ellipticity assumption on the spatial symbol of the hyperbolic operator, the authors obtain a result of strong well-posedness for corners problems with inhomogeneous initial conditions.

We give some more details about the mentioned previous energy estimates. By analogy with the natural energy estimate in the half space geometry [Kre70, the expected energy estimate for $u$ the solution of (1) is:

$$
\begin{aligned}
\sup _{t \geq 0} e^{-2 \gamma t}\|u(t, \cdot)\|_{L^{2}(\Omega)}^{2} & +\gamma \int_{0}^{\infty} e^{-2 \gamma t}\|u(t, \cdot)\|_{L^{2}(\Omega)}^{2} d t+\sum_{k=1}^{2} \int_{0}^{\infty} e^{-2 \gamma t}\left\|u_{\mid x_{k}=0}(t, \cdot)\right\|_{L^{2}\left(\partial \Omega_{k}\right)}^{2} d t \\
& \leq C\left(\left\|u_{0}\right\|_{L^{2}(\Omega)}^{2}+\frac{1}{\gamma} \int_{0}^{\infty} e^{-2 \gamma t}\|f(t, \cdot)\|_{L^{2}(\Omega)}^{2} d t+\sum_{k=1}^{2} \int_{0}^{\infty} e^{-2 \gamma t}\left\|g_{k}(t, \cdot)\right\|_{L^{2}\left(\partial \Omega_{k}\right)}^{2} d t\right)
\end{aligned}
$$

and, in our definition stability for difference schemes approximations of (1) means that some discretized version of 22) (see Definition 2.1 for a precise definition) holds for the solution of the scheme.

\subsection{Some results about strong well-posed and stability for the half space geom- etry}

Before to describe the obtained stability results, it is interesting to give a brief overview of the known results on this subject in the simpler geometry of the half space $\widetilde{\Omega}:=\left\{x=\left(x_{1}, x^{\prime}\right) \mid x_{1}>0, x^{\prime} \in \mathbb{R}^{d-1}\right\}$. The associated version of (1) reads:

$$
\left\{\begin{array}{l}
L(\partial) u=f, \text { in }[0, \infty[\times \widetilde{\Omega} \\
B u_{\mid x_{1}=0}=g_{1}, \text { on }[0, \infty[\times \partial \widetilde{\Omega} \\
u_{\mid t=0}=u_{0}, \text { on } \widetilde{\Omega}
\end{array}\right.
$$

and a finite difference scheme approximation of (3) is (for example for a one time step approximation) given by:

$$
\left\{\begin{array}{l}
U_{j}^{n+1}+Q U_{j}^{n}=\Delta t f_{j}^{n+1}, \text { for } n \geq 0, j_{1} \geq 1, j^{\prime} \in \mathbb{Z}^{d-1} \\
U_{j}^{n+1}+B^{0, j_{1}} U_{j}^{n}+B^{1, j_{1}} U_{j}^{n+1}=g_{j}^{n+1}, \text { for } n \geq 0, \geq 1-\ell_{1} \leq j_{1} \leq 0, j^{\prime} \in \mathbb{Z}^{d-1}, \\
U_{j}^{0}=u_{0, j}, \text { for } j_{1} \geq 1-\ell_{1}, j^{\prime} \in \mathbb{Z}^{d-1},
\end{array}\right.
$$

where $Q$ is a dicretization of the spatial differetiation in the interior, $B^{0, j_{1}}$ and $B^{1, j_{1}}$ are discretizations of the boundary condition $B$ and finally where $\ell_{1} \in \mathbb{N}$ is the stencil of the operator $Q$ in the $\left(-x_{1}\right)$-direction.

Compared to the corner geometry, the theory of semi-group well-posedness for (3) is much more elaborated. Semi-group well-posedness for (3) means existence and uniqueness of a solution $u$ which satisfies the energy estimate: there exists $C>0$ such that for all $\gamma>0$ we have

$$
\begin{aligned}
\sup _{t \geq 0} e^{-2 \gamma t}\|u(t, \cdot)\|_{L^{2}(\widetilde{\Omega})}^{2} & +\gamma \int_{0}^{\infty} e^{-2 \gamma t}\|u(t, \cdot)\|_{L^{2}(\widetilde{\Omega})}^{2} d t+\int_{0}^{\infty} e^{-2 \gamma t}\left\|u_{\mid x_{1}=0}(t, \cdot)\right\|_{L^{2}(\partial \widetilde{\Omega})}^{2} d t \\
& \leq C\left(\left\|u_{0}\right\|_{L^{2}(\widetilde{\Omega})}^{2}+\frac{1}{\gamma} \int_{0}^{\infty} e^{-2 \gamma t}\|f(t, \cdot)\|_{L^{2}(\widetilde{\Omega})}^{2} d t+\int_{0}^{\infty} e^{-2 \gamma t}\left\|g_{1}(t, \cdot)\right\|_{L^{2}(\partial \widetilde{\Omega})}^{2} d t\right) .
\end{aligned}
$$

And, from Kre70 and Rau72, we know that the initial boundary value problem in the half space (3) is semi-group well-posed if and only if the so-called uniform Kreiss-Lopatinskii condition is satisfied. This conditions means that in the normal modes analysis no stable mode satisfies the homogeneous boundary condition.

Semi-group stability for the finite difference scheme approximation (4) means (for example) that the 
solution of (4) satisfies the estimate: there exists $C$ such that for all $\gamma>0$, for all $\Delta t \in] 0,1]$

$$
\begin{aligned}
& \sup _{n \geq 0} \Delta x_{1} e^{-2 \gamma n \Delta t} \sum_{j_{1}=1-\ell_{1}}^{\infty}\left\|U_{j_{1},}^{n} .\right\|^{2}+\frac{\gamma}{\gamma \Delta t+1} \sum_{n \geq 0} \Delta t \Delta x_{1} e^{-2 \gamma n \Delta t} \sum_{j_{1}=1-\ell_{1}}^{\infty}\left\|U_{j_{1},}^{n} \cdot\right\|^{2}+\sum_{n \geq 0} \Delta t e^{-2 \gamma n \Delta t} \sum_{j_{1}=1-\ell_{1}}^{0}\left\|U_{j_{1},}^{n}\right\|^{2} \\
& \leq C\left(\sum_{j_{1}=1-\ell_{1}}^{\infty} \Delta x_{1}\left\|u_{0}\right\|^{2}+\frac{\gamma \Delta t+1}{\gamma} \sum_{n \geq 1} \Delta t \Delta x_{1} e^{-2 \gamma n \Delta t} \sum_{j_{1}=1-\ell_{1}}^{\infty}\left\|f_{j_{1},}^{n}\right\|^{2}\right. \\
&\left.+\sum_{n \geq 1} \Delta t e^{-2 \gamma n \Delta t} \sum_{j_{1}=1-\ell_{1}}^{0}\left\|g_{j_{1}, \cdot}^{n}\right\|^{2}\right), \quad(6)
\end{aligned}
$$

where the $\|\cdot\|$-norm is defined by: for $j^{\prime} \in \mathbb{Z}^{d-1}$,

$$
\|U .\|^{2}:=\prod_{k=2}^{d} \Delta x_{k} \sum_{j^{\prime} \in \mathbb{Z}^{d-1}}\left|U_{j^{\prime}}\right|^{2},
$$

and where the parameters $\Delta t, \Delta x_{k}, k=1, \ldots, d$ are the parameters of the cartesian discretization of $[0, \infty[\times \widetilde{\Omega}$. These parameters are assumed to satisfy some $C F L$ (COURANT-FRIEDRICHS-LEWY) condition (that is to say that the ratios $\lambda_{k}:=\frac{\Delta t}{\Delta x_{k}}$ are constant while $\left.\Delta t \downarrow 0\right)$.

Note that if ones formally takes the limit $\Delta t \downarrow 0$ in the stability estimate for $\left(U_{j}^{n}\right)$ then he recovers the energy estimate for $u$ that is (5). As a consequence the stability estimate for $\left(U_{j}^{n}\right)$ is just a discretized version of (5). Once again we have a full characterization of the difference schemes approximations that are strongly stable : the scheme (4) is strongly stable if and only it satisfies the so-called GKS (GustafssonKreiss-Sundström) condition (see [BGS72]). This condition is in some sense a discrete version of the uniform Kreiss-Lopatinskii condition.

The sketch of proof to establish the semi-group stability or the semi-group well-posed in the same and is based in two distinct substeps. In a first time the study is restricted to homogeneous initial conditions and we show the estimate (5) but without the control of the supremum in the left hand side (and also without the term $u_{0}$ in the right hand side). This estimate characterized all the problems which are call strongly well-posed (resp. strongly stable) in the setting of continous (resp. discrete) problems. More precisely this estimate in the continous setting reads: there exists $C>0$ such that for all $\gamma>0$

$$
\begin{aligned}
\gamma \int_{0}^{\infty} e^{-2 \gamma t}\|u(t, \cdot)\|_{L^{2}(\widetilde{\Omega})}^{2} d t+\int_{0}^{\infty} e^{-2 \gamma t}\left\|u_{\mid x_{1}=0}(t, \cdot)\right\|_{L^{2}(\partial \widetilde{\Omega})}^{2} d t & \\
& \leq C\left(\frac{1}{\gamma} \int_{0}^{\infty} e^{-2 \gamma t}\|f(t, \cdot)\|_{L^{2}(\widetilde{\Omega})}^{2} d t+\int_{0}^{\infty} e^{-2 \gamma t}\left\|g_{1}(t, \cdot)\right\|_{L^{2}(\partial \widetilde{\Omega})}^{2} d t\right),
\end{aligned}
$$

and: there exists $C>0$ such that for all $\gamma>0, \Delta t \in] 0,1]$

$$
\begin{aligned}
\frac{\gamma}{\gamma \Delta t+1} \sum_{n \geq 0} \Delta t \Delta x_{1} e^{-2 \gamma n \Delta t} \sum_{j_{1}=1-\ell_{1}}^{\infty}\left\|U_{j_{1},}^{n},\right\|^{2}+\sum_{n \geq 0} \Delta t e^{-2 \gamma n \Delta t} \sum_{j_{1}=1-\ell_{1}}^{0}\left\|U_{j_{1},}^{n},\right\|^{2} \\
\quad \leq C\left(+\frac{\gamma \Delta t+1}{\gamma} \sum_{n \geq 1} \Delta t \Delta x_{1} e^{-2 \gamma n \Delta t} \sum_{j_{1}=1-\ell_{1}}^{\infty}\left\|f_{j_{1},}^{n}\right\|^{2}+\sum_{n \geq 1} \Delta t e^{-2 \gamma n \Delta t} \sum_{j_{1}=1-\ell_{1}}^{0}\left\|g_{j_{1},}^{n}\right\|^{2}\right),
\end{aligned}
$$

in the discrete framework.

Then in a second time, this estimate characterizing the strong well-posedness (resp. strong stability) is extended to non homogeneous initial conditions and the supremum is added in the left hand side in view to obtain (resp. the discretized version of) (5). Once the estimate is demonstrated we tell that the problem is semi-group well-posed in the continous setting and semi-group stable in the discrete one. Let us note the important fact that in this second step, the main assumption is to assume that the considered problem 
is strongly well-posed or strongly stable (up to some possibly technical extra assumptions in the discrete framework).

In the continous setting the full characterization of strongly well-posed problems has been established in Kre70 and its extension to non homogeneous initial conditions establishing the semi-group well-posedness is due to Rau72 (we also refer to BG07 or CP81 for an overview/review of the proofs).

In the discrete setting, the first result showing strong stability for a finite difference scheme is due to BGS72] and has then been extended to more general finite difference schemes, for example, in [Cou09Cou11. About the semi-group stability of schemes with non zero initial conditions, the first result applies to one step in time finite difference schemes and is due to Wu95. The method of Wu95 has then been generelized in [CG11. An other result in the theory of semi-group stability for finite difference schemes with several time steps this time (but restricted to scalar equations) is obtained in Cou15.

\subsection{Generalization of semi-group stability results to corner domains}

In this article we give generalizations of the results contained in CG11 and Cou15 from the half space to the quarter space geometry. More precisely we show that if we assume that the finite difference scheme approximation is strongly stable (see Definition 2.1 for a precise definition) then a discretized version of (2) (see (22)-(24) can be obtained for all the finite difference schemes that we are able to deal with in the half space geometry.

As a consequence, the geometry in which the finite difference scheme is set does not prevent to go from strong stability to semi-group stability ${ }^{1}$

As the reader will see, our proofs follow the main steps of the proofs in CG11 and Cou15. This fact may seem to be surprising but it should not. Indeed the proofs in CG11 and Cou15 both relies on the introduction of an auxiliary problem. More precisely in [CG11, the authors first treate the case of one dimensional schemes. Then to generalize their result to multidimensional schemes they use partial Fourier transform in the tangential variables to recover the one dimensional case.

In the one dimensional setting, the auxiliary problem used in CG11 is the finite difference scheme (4) but with Dirichlet boundary conditions instead of the discretized boundary conditions involving $B^{0, j_{1}}$ and $B^{1, j_{1}}$. The auxiliary problem used in Cou15] in based on two discrete multipliers coming from the LerayGärding method to obtain a priori estimates for hyperbolic PDE (see [?]-[?]). Note that the existence of such multipliers was the starting point in the analysis of Rau72 to go from the strong well-posed to the semi-group stability for initial boundary value problems. Compared with the auxiliary problem used in CG11, the auxiliary problem of [Cou15] is defined on the full space $\left\{j \in \mathbb{Z}^{d}\right\}$ and thus it permits to use Laplace- partial Fourier transform (without any extension) to translate some energy estimates for the solution in terms of the symbol of the discretization operator.

Then the authors use the fact that each auxiliary finite difference scheme admits strictly dissipative boundary condition to show the semi-group stability from the strong stability.

In the continous setting it is known (see for example [ $[$ Ben15, Chapitre 4]) that strictly dissipative boundary conditions are suitable for corner problems as well as for problems in the half space. As a consequence, as far as strict dissipativity is concerned, the proofs for finite difference schemes in the half space should also operate for the quarter space geometry and it is effectively the case with sometimes really minor changes. An other important point in the generalization of Cou15 to the quarter space geometry is that the auxiliary problem is set in the full space. Consequently the use of the Laplace- partial Fourier transform (which is prohibited, without preliminary extension, in the quarter space geometry because there are two "normal" directions) also operates because we are in the full space.

This point will not be true anymore for the generalization of [CG11] for quarter spaces because the auxiliary problem will not be set in the full space. However we show that in that case it is possible to do the analysis of [CG11] and specifically the energy method directly for multidimensional schemes. So we will not

\footnotetext{
${ }^{1}$ In all the article to make the notations as simple as possible we restricted our subject to domains with only one two dimensional corner. However all the results extended to multi-dimensional corners and/or to domains with several corners.
} 
have to perform any partial Fourier transform and the result extend to the quarter space geometry.

Of course the main assumption in both of the generalization is that the finite difference scheme for the corner problem is strongly stable. In the author knowledge there is no result concerning the full characterization of strongly stable schemes in corner domains in the litterature. Moreover in the author opinion this question could be a challenging one. What is clear is that imposing that each finite difference scheme in the half spaces $\left\{j_{1} \geq 1-\ell_{1},\left(j_{2}, j^{\prime}\right) \in \mathbb{Z}^{d-1}\right\}$ and $\left\{j_{1} \in \mathbb{Z}, j_{2} \geq 1-\ell_{2}, j^{\prime} \in \mathbb{Z}^{d-2}\right\}$ satisfies the GKS condition will be necessary. However the study of Osh73 for continous problems tells us that a new condition will be needed. In analogy with the half space geometry, that seems to be a reasonable conjecture is that to characterize strong stability in corner domains a discretized version of this condition will also be necessary.

\subsection{Organization of the article}

The paper is organized as follows. In Section 2 we introduce the notations and some definitions, in particular we give some new definitions needed to deal with the corner geometry. Then in Section 3 we state the assumptions and the main results. At last Sections 4 and 5 are devoted to the proofs of each generalization.

\section{Finite difference schemes and corner}

\subsection{General notations and definitions}

In all what follows we use the short hand notation $\llbracket \cdot, \cdot \rrbracket$ for the "intervals of integers", more precisely for $a, b \in \mathbb{R}$ we define $\llbracket a, b \rrbracket:=[a, b] \cap \mathbb{Z}$.

To describe the finite difference scheme that we will consider we define the following subsets of $\mathbb{Z}^{2}$, for $j=\left(j_{1}, j_{2}\right) \in \mathbb{Z}^{2}$ let:

$$
\begin{aligned}
\mathscr{I}:=\left\{j \in \mathbb{Z}^{2} \mid j_{1}, j_{2} \geq 1\right\}, \quad \mathscr{C}:=\left\{j \in \mathbb{Z}^{2} \mid j_{1} \in \llbracket 1-\ell_{1}, 0 \rrbracket, j_{2} \in \llbracket 1-\ell_{2}, 0 \rrbracket\right\} \\
\mathscr{B}_{1}:=\left\{j \in \mathbb{Z}^{2} \mid j_{1} \in \llbracket 1-\ell_{1}, 0 \rrbracket, 1 \leq j_{2}\right\}, \quad \mathscr{B}_{2}:=\left\{j \in \mathbb{Z}^{2} \mid 1 \leq j_{1}, j_{2} \in \llbracket 1-\ell_{2}, 0 \rrbracket\right\},
\end{aligned}
$$

where $\ell_{1}$ (resp. $\ell_{2}$ ) is a fixed positive integer (that will correspond to the number of space steps of the scheme towards the "left" (resp. "bottom")).

The set $\mathscr{I}$ has to be understood as the discretization of the interior of $\Omega, \mathscr{B}_{1}$ (resp. $\mathscr{B}_{2}$ ) as the discretization of the boundary $\partial \Omega_{1}$ (resp. $\partial \Omega_{2}$ ) and finally $\mathscr{C}$ is a discretization of the corner of $\Omega$. Finally, the full set of resolution $\mathscr{R}$ is defined by

$$
\mathscr{R}:=\mathscr{I} \cup \mathscr{B}_{1} \cup \mathscr{B}_{2} \cup \mathscr{C} \text {. }
$$

To state our definition of strong stability we introduce the extended discretizations of the traces $\mathscr{B}_{1}$ and $\mathscr{B}_{2}$ defined by:

$$
\overline{\mathscr{B}}_{1}:=\left\{j \in \mathbb{Z}^{2} \mid j_{1} \in \llbracket 1-\ell_{1}, r_{1} \rrbracket, 1-\ell_{2} \leq j_{2}\right\}, \overline{\mathscr{B}}_{2}:=\left\{j \in \mathbb{Z}^{2} \mid 1-\ell_{1} \leq j_{1}, j_{2} \in \llbracket 1-\ell_{2}, r_{2} \rrbracket\right\},
$$

where once again $r_{1}$ (resp. $r_{2}$ ) is a fixed positive integer (that will correspond to the number of space steps of the scheme towards the "right" (resp. "top")).

Let $\Delta x_{1}, \Delta x_{2}, \ldots \Delta x_{d}>0$ be the space steps of discretization, we define $\Delta x:=\Delta x_{1} \Delta x_{2}$, and let $\Delta t$ be the time step discretization. In a classical setting let us assume that $\Delta t, \Delta x_{1}, \ldots, \Delta x_{d}$ are related by the CFL numbers which are defined by $\lambda_{k}:=\frac{\Delta t}{\Delta x_{k}}$ for $k \in \llbracket 1, d \rrbracket$. Let us recall that the $\lambda_{k}$ are kept constant as $\Delta t \downarrow 0$. Note that it implies, in particular, that for all $k_{1}, k_{2} \in \llbracket 1, d \rrbracket$ we have $\Delta x_{k_{1}} \sim \Delta x_{k_{2}}$.

We introduce the following weighted norm on $\ell^{2}\left(\mathbb{Z}^{d}\right)$. Let $\mathfrak{I} \subseteq \mathbb{Z}^{2}$ and $u \in \ell^{2}\left(\mathfrak{I} \times \mathbb{Z}^{d-2}\right)$ we define:

$$
\|u\|_{\mathfrak{I}}^{2}:=\left(\prod_{k=3}^{d} \Delta x_{k}\right)\|u\|_{\ell^{2}\left(\mathfrak{I} \times \mathbb{Z}^{d-2}\right)}^{2} \text {, and }\|u\|_{\mathfrak{I}}^{2}:=\Delta x_{1} \Delta x_{2}\|u\|_{\mathfrak{I}}^{2} .
$$




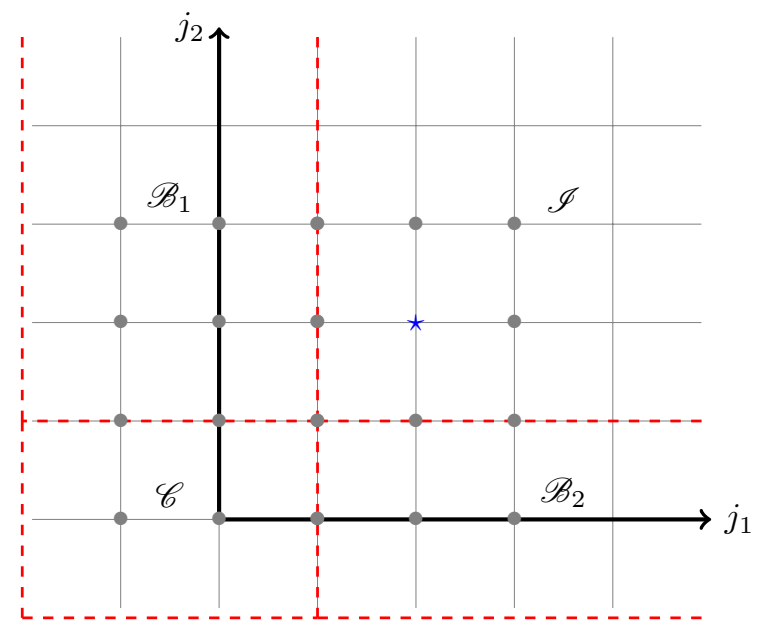

Figure 1: The set of resolution and the dependency set of $U_{2,2}$ for $\ell_{1}=3, \ell_{2}=2$ and $r_{1}=r_{2}=1$.

We also denote $\langle\cdot, \cdot\rangle_{\mathfrak{I}}\left(\right.$ resp. $\left.\langle\langle\cdot, \cdot\rangle\rangle_{\mathfrak{I}}\right)$ the scalar product associated to $\|\cdot\|_{\mathfrak{I}}\left(\right.$ resp. $\left.\|\cdot\|_{\mathfrak{I}}^{2}\right)$.

The finite difference scheme approximation of (1) that we are considering reads:

$$
\begin{cases}\sum_{\sigma=0}^{s+1} Q^{\sigma} U_{j}^{n+\sigma}=\Delta t f_{j}^{n+s+1}, & \text { for } j \in \mathscr{I} \times \mathbb{Z}^{d-2}, n \geq 0, \\ U_{j}^{n+s+1}+\sum_{\sigma=0}^{s+1} B_{1}^{\sigma, j_{1}} U_{j}^{n+\sigma}=g_{1, j}^{n+s+1}, & \text { for } j \in \mathscr{B}_{1} \times \mathbb{Z}^{d-2}, n \geq 0, \\ U_{j}^{n+s+1}+\sum_{\sigma=0}^{s+1} B_{2}^{\sigma, j_{2}} U_{j}^{n+\sigma}=g_{2, j}^{n+s+1}, & \text { for } j \in \mathscr{B}_{2} \times \mathbb{Z}^{d-2}, n \geq 0, \\ U_{j}^{n+s+1}+\sum_{\sigma=0}^{s+1} C^{\sigma, j} U_{j}^{n+\sigma}=h_{j}^{n+s+1}, & \text { for } j \in \mathscr{C} \times \mathbb{Z}^{d-2}, n \geq 0, \\ U_{j}^{n}=u_{n, j}, & \text { for } j \in \mathscr{R} \times \mathbb{Z}^{d-2}, n \in \llbracket 0, s \rrbracket .\end{cases}
$$

Note that $10 p$ has $s+1$ time steps. The operator $Q^{\sigma}$ appearing in the first equation of 10$]$ is defined by:

$$
Q^{\sigma}:=\sum_{\mu_{1}=-\ell_{1}}^{r_{1}} \sum_{\mu_{2}=-\ell_{2}}^{r_{2}} \sum_{\mu^{\prime}=-\ell^{\prime}}^{r^{\prime}} A^{\sigma, \mu} \mathbf{T}_{1}^{\mu_{1}} \mathbf{T}_{2}^{\mu_{2}} \mathbf{T}^{\mu^{\prime}}
$$

where $\mu:=\left(\mu_{1}, \mu_{2}, \mu^{\prime}\right) \in \mathbb{N}^{d}$, the coefficients $A^{\mu} \in \mathbf{M}_{n \times n}(\mathbb{R})$ and where for $k \in \llbracket 1,2 \rrbracket, \mathbf{T}_{k}^{\mu_{k}}$ (resp. $\mathbf{T}^{\prime \mu^{\prime}}$ ) denotes the $\mu_{k}$ (resp. $\left.\mu^{\prime}\right)$-shift operator, that is:

$$
\forall u \in \ell^{2}\left(\mathbb{Z}^{d}\right),\left(\mathbf{T}_{1}^{\mu_{1}} u\right)_{j}:=u_{j_{1}+\mu_{1}, j_{2}, j^{\prime}},\left(\mathbf{T}_{2}^{\mu_{2}} u\right)_{j}:=u_{j_{1}, j_{2}+\mu_{2}, j^{\prime}} \text { and }\left(\mathbf{T}^{\prime \mu^{\prime}} u\right)_{j}:=u_{j_{1}, j_{2}, j^{\prime}+\mu^{\prime}} .
$$

Also note that in 11] we used the short hand notation: for $\ell^{\prime}, r^{\prime} \in \mathbb{N}^{d-2}$,

$$
\sum_{\mu^{\prime}=-\ell^{\prime}}^{r^{\prime}}:=\sum_{k=3}^{d-2} \sum_{\mu_{k}=-\ell_{k}}^{r_{k}} .
$$

Thus, in view of its definition, the scheme $(10)$ has stencil $\ell_{1}+r_{1}$ in the $j_{1}$-direction and $\ell_{2}+r_{2}$ in the $j_{2}$-direction. So to compute the sequence $\left(U_{j}^{n}\right)_{j \in \mathscr{I}}$ it is needed to know the boundary values $\left(U_{j}^{n}\right)_{j \in \mathscr{B}_{1} \cup \mathscr{B}_{2}}$. This was expected from the analysis of finite difference schemes in the half space. But, and it is a new fact induced by the quarter space geometry, we also need the corner values $\left(U_{j}^{n}\right)_{j \in \mathscr{C}}$ (see (8)-77) for a definition of this sets). Also note that in this formulation, the finite difference scheme (10) can be explicit or implicit in time.

A new feature for finite difference scheme in corner domains is that, if we have computed the solution $\left(U_{j}^{n}\right)_{j \in \mathscr{R}}$ at some time $n$ then, the order of computation of the $U_{j}^{n+1}$ is not as canonical as in the half space geometry. Indeed for finite difference schemes in the half space the only possible way to compute $\left(U_{j}^{n+1}\right)$ 
from $\left(U_{j}^{n}\right)$ is to determine the $U_{j}^{n+1}$ for $j$ in the interior and then to compute the $U_{j}^{n+1}$ for $j$ in the discretization of the boundary. This determines $\left(U_{j}^{n+1}\right)$ and the order also of resolution of the scheme in a unique way.

In corner domains we always have to determine first the $U_{j}^{n+1}$ for $j \in \mathscr{I}$. But we have some degrees of freedom in the order of determination of the $U_{j}^{n+1}$ for $j \in \mathscr{B}_{1} \cup \mathscr{B}_{2} \cup \mathscr{C}$. These degrees of freedom lead to different expressions of the boundary and corner operators $B_{1}^{\sigma, j_{1}}, B_{2}^{\sigma, j_{2}}$ and $C^{\sigma, j_{1}, j_{2}}$. Some of this several possible expressions are described in paragraph 2.2

We conclude this section by the definition of strongly stable finite difference schemes in the quarter space:

Definition 2.1 (Strong stability) We say that the difference scheme 10 is strongly stable for homogeneous initial conditions if there exists $C>0$ such that for all $\gamma>0$ and $\Delta t \in] 0,1]$, the solution $\left(U_{j}^{n}\right)$ of $(10)$ with $u_{0, j} \equiv 0$ satisfies the estimat ${ }^{2}$ :

$$
\begin{gathered}
\frac{\gamma}{\gamma \Delta t+1} \sum_{n \geq s+1} \Delta t e^{-2 \gamma n \Delta t}\left\|U^{n}\right\|_{\mathscr{R}}^{2}+\sum_{k=1}^{2} \sum_{n \geq s+1} \Delta t \Delta x_{3-k} e^{-2 \gamma n \Delta t}\left\|U^{n}\right\|_{\mathscr{B}_{k}}^{2} \leq \\
C\left(\frac{\gamma \Delta t+1}{\gamma} \sum_{n \geq s+1} \Delta t e^{-2 \gamma n \Delta t}\left\|f^{n}\right\|_{\mathscr{I}}^{2}+\sum_{k=1}^{2} \sum_{n \geq s+1} \Delta t \Delta x_{3-k} e^{-2 \gamma n \Delta t}\left\|g_{k}^{n}\right\|_{\mathscr{B}_{k}}^{2}+\sum_{n \geq s+1} \Delta t \Delta x_{2} e^{-2 \gamma n \Delta t}\left\|h^{n}\right\|_{\mathscr{C}}^{2}\right) .
\end{gathered}
$$

Before we turn to the statement of our main result it may be interesting to give some comments about the notion of strong stability given in Definition 2.1. Indeed many definitions of stability are possible, and to the author knowledge, any definition of strong stability has been proposed for difference schemes for a boundary value problem in the quarter space. Remark that when one takes the limit $\Delta t \downarrow 0$ in $(12)$ then he (formally) recovers the expected energy estimate for initial boundary value problems in the quarter space (2).

\subsection{Boundary and corner conditions}

In this paragraph we give several possible expressions for the boundary and corner operators $B_{1}^{\sigma, j_{1}}, B_{2}^{\sigma, j_{2}}$ and $C^{\sigma, j_{1}, j_{2}}$ appearing in 10 and we then describe the influence of these expressions on the order of determination of the $U_{j}^{n+1}$ for $j \in \mathscr{B}_{1} \cup \mathscr{B}_{2} \cup \mathscr{C}$.

The first possible choice is the simplest one. It is also the one that most looks like the boundary conditions in the half space. We first define for the boundary operators:

$$
\begin{aligned}
& \text { for } j \in \mathscr{B}_{1}, B_{1}^{\sigma, j_{1}}:=\sum_{\mu_{1}=0}^{q_{11}} \sum_{\mu_{2}=0}^{q_{12}} \sum_{\mu^{\prime}=-q_{1}^{\prime}}^{q_{1}^{\prime}} B_{1}^{\sigma, \mu, j_{1}} \mathbf{T}_{1}^{\mu_{1}} \mathbf{T}_{2}^{\mu_{2}} \mathbf{T}^{\prime \mu^{\prime}} \\
& \text { for } j \in \mathscr{B}_{2}, \quad B_{2}^{\sigma, j_{2}}:=\sum_{\mu_{1}=0}^{q_{21}} \sum_{\mu_{2}=0}^{q_{22}} \sum_{\mu^{\prime}=-q_{2}^{\prime}}^{q_{2}^{\prime}} B_{2}^{\sigma, \mu, j_{2}} \mathbf{T}_{1}^{\mu_{1}} \mathbf{T}_{2}^{\mu_{2}} \mathbf{T}^{\prime \mu^{\prime}}
\end{aligned}
$$

where $q_{11}, q_{12}, q_{21}$ and $q_{22}$ are fixed positive integers, $q_{1}^{\prime}, q_{2}^{\prime} \in \mathbb{N}^{d-2}$ and where the $B_{1}^{\sigma, \mu, j_{1}}, B_{2}^{\sigma, \mu, j_{2}}$ are fixed matrices in $\mathbf{M}_{n \times n}(\mathbb{R})$. And we then define the corner operator by:

$$
\text { for } j \in \mathscr{C}, C^{\sigma, j_{1}, j_{2}}:=\sum_{\mu_{1}=0}^{c_{1}} \sum_{\mu_{2}=0}^{c_{2}} \sum_{\mu^{\prime}=-c^{\prime}}^{c^{\prime}} C^{\sigma, \mu, j_{1}, j_{2}} \mathbf{T}_{1}^{\mu_{1}} \mathbf{T}_{2}^{\mu_{2}} \mathbf{T}^{\prime \mu^{\prime}},
$$

for $c_{1}, c_{2}$ two positive fixed integers and $c^{\prime} \in \mathbb{N}^{d-2}$. The coefficients $C^{\sigma, \mu, j_{1}, j_{2}}$ are fixed matrices in $\mathbf{M}_{n \times n}(\mathbb{R})$.

\footnotetext{
${ }^{2}$ Let us remark that by definition of the CFL numbers $\lambda_{1}$ and $\lambda_{2}$ we have $\Delta x_{1} \sim \Delta x_{2}$ and as a consequence one can equivalently use 12 with the last term in the right hand side changed by $\sum_{n \geq s+1} \Delta t \Delta x_{1} e^{-2 \gamma n \Delta t}\left\|h^{n}\right\|_{\mathscr{C}}^{2}$ (or even $\left.\sum_{n \geq s+1} \Delta t^{2} e^{-2 \gamma n \Delta t}\left\|h^{n}\right\|_{\mathscr{C}}^{2}\right)$ as an estimate for strongly stable finite difference schemes.
} 
With these definitions the terms $U_{j}^{n+1}$ for $j \in \mathscr{B}_{1} \cup \mathscr{B}_{2} \cup \mathscr{C}$ only depend on the $U_{j}^{n}$ for $j \in \mathscr{R}$ and the $U_{j}^{n+1}$ for $j \in \mathscr{I}$. So the $U_{j}^{n+1}$ for $j \in \mathscr{B}_{1} \cup \mathscr{B}_{2} \cup \mathscr{C}$ can be determined in any order.

A second possibility is to keep (13) and 14 for the equations defining the boundary operators but to change (15) by:

$$
\text { for } \begin{aligned}
j \in \mathscr{C}, C^{\sigma, j_{1}, j_{2}} & :=\sum_{\mu^{\prime}=-c^{\prime}}^{c^{\prime}}\left(\sum_{\mu_{1}=0}^{c_{1}} \sum_{\mu_{2}=0}^{c_{2}} C^{\sigma, \mu, j_{1}, j_{2}} \mathbf{T}_{1}^{\mu_{1}} \mathbf{T}_{2}^{\mu_{2}}\right. \\
& \left.+\sum_{\mu_{1}=-\ell_{1}}^{-1} \sum_{\mu_{2}=0}^{c_{12}} C_{\mathscr{B}_{1}}^{\sigma, \mu, j_{1}, j_{2}} \mathbf{T}_{1}^{\mu_{1}} \mathbf{T}_{2}^{\mu_{2}}+\sum_{\mu_{1}=0}^{c_{21}} \sum_{\mu_{2}=-\ell_{2}}^{-1} C_{\mathscr{B}_{2}}^{\sigma, \mu, j_{1}, j_{2}} \mathbf{T}_{1}^{\mu_{1}} \mathbf{T}_{2}^{\mu_{2}}\right) \mathbf{T}^{\prime \mu^{\prime}},
\end{aligned}
$$

where $c_{12}$ and $c_{21}$ are fixed integers and $C_{\mathscr{B}_{1}}^{\sigma, \mu, j_{1}, j_{2}}, C_{\mathscr{B}_{2}}^{\sigma, \mu, j_{1}, j_{2}}$ are fixed matrices in $\mathbf{M}_{n \times n}(\mathbb{R})$. With this new definition of $C^{s+1, j_{1}, j_{2}}, C^{s+1, j_{1}, j_{2}} U_{1,1}^{n+1}$ now involves some terms of the discretized boundaries $\mathscr{B}_{1}$ and $\mathscr{B}_{2}$ (more precisely the $U_{j}^{n+1}$ for $\left.j \in\left(\llbracket 1-\ell_{1}, 0 \rrbracket \times \llbracket 1,1+c_{12} \rrbracket\right) \cup\left(\llbracket 1,1+c_{21} \rrbracket \times \llbracket 1-\ell_{2}, 0 \rrbracket\right)\right)$ and thus it is needed to determine the $U_{j}^{n+1}$ for $j \in \mathscr{B}_{1} \cup \mathscr{B}_{2}$ before to determine the $U_{j}^{n+1}$ for $j \in \mathscr{C}$.

The last possibility that we will describe here is to go back to 15 for the equation defining the corner operator and to change the equations defining the boundary operators by:

$$
\text { for } j_{1} \in \llbracket 1-\ell_{1}, 0 \rrbracket, \quad B_{1}^{\sigma, j_{1}}:=\sum_{\mu^{\prime}=-q_{1}^{\prime}}^{q_{1}^{\prime}}\left(\sum_{\mu_{1}=0}^{q_{11}} \sum_{\mu_{2}=0}^{q_{12}} B_{1}^{\sigma, \mu, j_{1}} \mathbf{T}_{1}^{\mu_{1}} \mathbf{T}_{2}^{\mu_{2}}+\sum_{\mu_{1}=-\ell_{1}}^{-1} \sum_{\mu_{2}=-\ell_{2}}^{-1} B_{1, \mathscr{C}}^{\sigma, \mu, j_{1}} \mathbf{T}_{1}^{\mu_{1}} \mathbf{T}_{2}^{\mu_{2}}\right) \mathbf{T}^{\prime \mu^{\prime}}
$$

for $j_{2} \in \llbracket 1-\ell_{2}, 0 \rrbracket, \quad B_{2}^{\sigma, j_{2}}:=\sum_{\mu^{\prime}=-q_{2}^{\prime}}^{q_{2}^{\prime}}\left(\sum_{\mu_{1}=0}^{q_{21}} \sum_{\mu_{2}=0}^{q_{22}} B_{2}^{\sigma, \mu, j_{2}} \mathbf{T}_{1}^{\mu_{1}} \mathbf{T}_{2}^{\mu_{2}}++\sum_{\mu_{1}=-\ell_{1}}^{-1} \sum_{\mu_{2}=-\ell_{2}}^{-1} B_{2, \mathscr{C}}^{\sigma, \mu, j_{2}} \mathbf{T}_{1}^{\mu_{1}} \mathbf{T}_{2}^{\mu_{2}}\right) \mathbf{T}^{\mu^{\prime}}$,

where $B_{1, \mathscr{C}}^{\sigma, \mu, j_{1}}, B_{2, \mathscr{C}}^{\sigma, \mu, j_{2}} \in \mathbf{M}_{n \times n}(\mathbb{R})$. In that case, the boundary terms depend of the corner terms and thus the boundary terms have to be computed after the corner ones.

We summarize the previous discussion in the following definition:

Definition 2.2 We say that the finite difference scheme approximation (10):

$\diamond$ admits decoupled boundary and corner conditions if the boundary operators are given by (13) and (14) and if the corner operator is given by 115 ;

$\diamond$ is traces to corner if the boundary operators are given by (13) and (14) and if the corner operator is given by 16 ;

$\diamond$ is corner to traces if the boundary operators are given by (17) and (18) and if the corner operator is given by 15 .

\section{Main results}

In all this article we will assume that the boundaries $\mathscr{B}_{1}$ and $\mathscr{B}_{2}$ are non characteristic for the scheme 10 . In view to state this assumption, let us introduce the following "tangential" operators from $\ell^{2}(\mathbb{Z})$ to $\ell^{2}(\mathbb{Z})$ : for $z \in \mathbb{C}$ and

$$
\begin{aligned}
& \text { for } \mu_{1} \in \llbracket-\ell_{1}, r_{1} \rrbracket, \mathbb{A}_{1}^{\mu_{1}}(z):=\sum_{\sigma=0}^{s+1} z^{\sigma} \sum_{\mu_{2}=-\ell_{2}}^{r_{2}} \sum_{\mu^{\prime}=-\ell^{\prime}}^{r^{\prime}} A^{\sigma, \mu} \mathbf{T}_{2}^{\mu_{2}} \mathbf{T}^{\mu^{\prime}}, \\
& \text { for } \mu_{2} \in \llbracket-\ell_{2}, r_{2} \rrbracket, \mathbb{A}_{2}^{\mu_{2}}(z):=\sum_{\sigma=0}^{s+1} z^{\sigma} \sum_{\mu_{1}=-\ell_{1}}^{r_{1}} \sum_{\mu^{\prime}=-\ell^{\prime}}^{r^{\prime}} A^{\sigma, \mu} \mathbf{T}_{1}^{\mu_{1}} \mathbf{T}^{\mu^{\prime}} .
\end{aligned}
$$

As already mentioned in the introduction, our first semi-group stability result holds for explicit with only one time step finite differences schemes (but with an arbitrary number of equations). In (10) we thus set 
$s=0$ and $Q^{1}=I$ to obtain (setting also $Q^{0}:=Q$ ):

$$
\begin{cases}U_{j}^{n+1}+Q U_{j}^{n}=\Delta t f_{j}^{n+1}, & \text { for } j \in \mathscr{I} \times \mathbb{Z}^{d-2}, n \geq 0, \\ U_{j}^{n+1}+B_{1}^{0, j_{1}} U_{j}^{n}+B_{1}^{1, j_{1}} U_{j}^{n+1}=g_{1, j}^{n+1}, & \text { for } j \in \mathscr{B}_{1} \times \mathbb{Z}^{d-2}, n \geq 0, \\ U_{j}^{n+1}+B_{2}^{0, j_{2}} U_{j}^{n}+B_{2}^{1, j_{2}} U_{j}^{n+1}=g_{2, j}^{n+1}, & \text { for } j \in \mathscr{B}_{2} \times \mathbb{Z}^{d-2}, n \geq 0, \\ U_{j}^{n+1}+C^{0, j_{1}, j_{2}} U_{j}^{n}+C^{0, j_{1}, j_{2}} U_{j}^{n}=h_{j}^{n+1}, & \text { for } j \in \mathscr{C} \times \mathbb{Z}^{d-2}, n \geq 0, \\ U_{j}^{0}=u_{0, j}, & \text { for } j \in \mathscr{R} \times \mathbb{Z}^{d-2} .\end{cases}
$$

In this framework the operators defined in $(19)$ and $(20)$ becomes:

$$
\mathbb{A}_{1}^{\mu_{1}}(z):=z \delta_{\mu_{1}=0}+\sum_{\mu_{2}=-\ell_{2}}^{r_{2}} \sum_{\mu^{\prime}=-\ell^{\prime}}^{r^{\prime}} A^{0, \mu} \mathbf{T}_{2}^{\mu_{2}} \mathbf{T}^{\prime \mu^{\prime}}, \text { and } \mathbb{A}_{2}^{\mu_{2}}(z):=z \delta_{\mu_{2}=0}+\sum_{\mu_{1}=-\ell_{1}}^{r_{1}} \sum_{\mu^{\prime}=-\ell^{\prime}}^{r^{\prime}} A^{0, \mu} \mathbf{T}_{1}^{\mu_{1}} \mathbf{T}^{\prime \mu^{\prime}},
$$

And, to save some notations, we also define $\mathbb{A}_{k}^{\mu_{k}}:=\mathbb{A}_{k}^{\mu_{k}}(1)$. The first non characteristicity assumption then reads:

Assumption 3.1 We assume that there exist two constants $c_{1}, c_{2}>0$ such that:

$$
\forall u \in \ell^{2}\left(\mathbb{Z}^{d}\right),\left\|\mathbb{A}_{1}^{r_{1}} u\right\| \geq c_{1}\|u\|, \text { and }\left\|\mathbb{A}_{2}^{r_{2}} u\right\| \geq c_{1}\|u\| .
$$

As in CG11] we assume that the operator of discretization in the interior $Q$ does not increase the $\ell^{2}$-norm of the solution.

Assumption 3.2 We assume that for all $u \in \ell^{2}\left(\mathbb{Z}^{d}\right)$, we have $\|Q u\| \leq\|u\|$.

Under these assumptions the generalization of [CG11 to the corner space geometry is the following:

Theorem 3.1 Under Assumptions 3.1 and 3.2, assume that the difference scheme approximation (21) is strongly stable in the sense of Definition 2.1 and finally $y^{3}$ assume that $r_{1}, r_{2} \geq 1$, then (21) is also semi-group stable. More precisely, there exists $C>0$ such that for all $\gamma>0$ and $\Delta t \in] 0,1]$ the solution of (21) satisfies the estimate:

$$
\begin{aligned}
\sup _{n \geq 0} e^{-2 \gamma n \Delta t}\left\|U^{n}\right\|_{\mathscr{R}}^{2} & +\frac{\gamma}{\gamma \Delta t+1} \sum_{n \geq 0} \Delta t e^{-2 \gamma n \Delta t}\left\|U^{n}\right\|_{\mathscr{R}}^{2}+\sum_{k=1}^{2} \sum_{n \geq 0} \Delta t \Delta x_{3-k} e^{-2 \gamma n \Delta t}\left\|U^{n}\right\|_{\mathscr{B}_{k}}^{2} \leq \\
& C\left(\left\|u_{0}\right\|_{\mathscr{R}}^{2}+\frac{\gamma \Delta t+1}{\gamma} \sum_{n \geq 1} \Delta t \Delta x e^{-2 \gamma n \Delta t}\left\|f^{n}\right\|_{\mathscr{I}}^{2}\right. \\
& \left.+\sum_{k=1}^{2} \sum_{n \geq 1} \Delta t \Delta x_{3-k} e^{-2 \gamma n \Delta t}\left\|g_{1}^{n}\right\|_{\mathscr{B}_{k}}^{2}+\sum_{n \geq 1} \Delta t e^{-2 \gamma n \Delta t}\left\|h^{n}\right\|_{\mathscr{C}}^{2}\right) .
\end{aligned}
$$

Our second main result is the generalization of [Cou15] for corner domains. This result is thus, on the one hand, restricted to scalar equations (that is $n=1$ ) but, on the other hand, it can be applied to finite difference schemes with several time steps. To stress that in this framework we are dealing with scalar equations we rewrite the coefficients defining (10), that is $Q^{\sigma, \mu}, B_{1}^{\sigma, \mu, j_{1}}, B_{2}^{\sigma, \mu, j_{2}}$ and $C^{\sigma, \mu, j}$, and the solution $\left(U_{j}^{n}\right)$ of 10 with lowercase letters. We thus write:

$$
\begin{cases}\sum_{\sigma=0}^{s+1} Q^{\sigma} u_{j}^{n+\sigma}=\Delta t f_{j}^{n+s+1}, & \text { for } j \in \mathscr{I} \times \mathbb{Z}^{d-2}, n \geq 0, \\ u_{j}^{n+s+1}+\sum_{\sigma=0}^{s+1} B_{1}^{\sigma, j_{1}} u_{j}^{n+\sigma}=g_{1, j}^{n+s+1}, & \text { for } j \in \mathscr{B}_{1} \times \mathbb{Z}^{d-2}, n \geq 0, \\ u_{j}^{n+s+1}+\sum_{\sigma=0}^{s+1} B_{2}^{\sigma, j_{2}} u_{j}^{n+\sigma}=g_{2, j}^{n+s+1}, & \text { for } j \in \mathscr{B}_{2} \times \mathbb{Z}^{d-2}, n \geq 0, \\ u_{j}^{n+s+1}+\sum_{\sigma=0}^{s+1} C^{\sigma, j_{1}, j_{2}} u_{j}^{n+\sigma}=h_{j}^{n+s+1}, & \text { for } j \in \mathscr{C} \times \mathbb{Z}^{d-2}, n \geq 0, \\ u_{j}^{n}=u_{n, j}, & \text { for } j \in \mathscr{R} \times \mathbb{Z}^{d-2}, n \in \llbracket 0, s \rrbracket .\end{cases}
$$

\footnotetext{
${ }^{3}$ If $r_{1}$ or $r_{2}$ equals zero then Theorem 3.1 remains true. This fact is straightforward direct use of the arguments of CG11] to treat the case $r_{1}=0$ that we will not reproduce here.
} 
The semi-group stability result then needs extra (or just some modifications) of Assumptions 3.1 and 3.2

The first assumption is made to ensure the solvability of (23) in the case that it defines an implicit (in time) scheme.

Assumption 3.3 The operator $Q^{s+1}$ appearing in $(23)$ is an isomorphism on $\ell^{2}\left(\mathbb{Z}^{d}\right)$. Moreover, for all source terms $f_{j} \in \ell^{2}\left(\mathscr{I} \times \mathbb{Z}^{d-2}\right), g_{1, j} \in \ell^{2}\left(\mathscr{B}_{1} \times \mathbb{Z}^{d-2}\right), g_{2, j} \in \ell^{2}\left(\mathscr{B}_{2} \times \mathbb{Z}^{d-2}\right)$ and $h \in \ell^{2}\left(\mathscr{C} \times \mathbb{Z}^{d-2}\right)$, the finite difference scheme:

$$
\begin{cases}Q^{s+1} u_{j}=f_{j}, & \text { for } j \in \mathscr{I} \times \mathbb{Z}^{d-2}, \\ u_{j}+B_{1}^{s+1, j_{1}} u_{j}=g_{1, j}, & \text { for } j \in \mathscr{B}_{1} \times \mathbb{Z}^{d-2}, \\ u_{j}+B_{2}^{s+1, j_{2}} u_{j}=g_{2, j}, & \text { for } j \in \mathscr{B}_{2} \times \mathbb{Z}^{d-2}, \\ u_{j}+C^{s+1, j_{1}, j_{2}} u_{j}=h_{j}, & \text { for } j \in \mathscr{C} \times \mathbb{Z}^{d-2},\end{cases}
$$

admits a unique solution $\left(u_{j}\right) \in \ell^{2}\left(\mathscr{R} \times \mathbb{Z}^{d-2}\right)$.

We also assume the following modifications of Assumptions 3.2 and 3.1 .

Assumption 3.4 For all $\eta:=\left(\eta_{1}, \eta_{2}, \eta^{\prime}\right) \in \mathbb{R}^{d}$, the equation

$$
\sum_{\sigma=0}^{s+1} \widehat{Q^{\sigma}}\left(e^{i \eta_{1}}, e^{i \eta_{2}}, \ldots, e^{i \eta_{d}}\right) z^{\sigma}=0, \text { where } \widehat{Q^{\sigma}}(\kappa):=\sum_{k=1}^{d} \sum_{\mu_{k}=-\ell_{k}}^{r_{k}} a^{\sigma, \mu} \kappa_{k}^{\mu_{k}},
$$

admits $s+1$ simple roots $z_{0}, \ldots, z_{d}$ satisfying that for all $k \in \llbracket 0, d \rrbracket,\left|z_{k}\right| \leq 1$.

Let us recall that Assumption 3.4 implies that the finite difference scheme associated to the Cauchy problem is strongly stable (see ()).

Our last assumption is a modification of Assumption 3.1

Assumption 3.5 For $z \in \mathbb{C}, \eta^{\prime} \in \mathbb{R}^{d-2} ; k \in \llbracket 1,2 \rrbracket, \mu_{k} \in \llbracket-\ell_{k}, r_{k} \rrbracket$ and $\eta_{3-k} \in \mathbb{R}$ we define:

$$
a_{k}^{\mu_{k}}\left(z, \eta^{\prime}, \eta_{3-k}\right):=\sum_{\sigma=0} z^{\sigma} \sum_{\mu_{3-k}=-\ell_{3-k}}^{r_{3-k}} a^{\sigma, \mu} e^{i \eta_{3-k} \mu_{3-k}} e^{i \eta^{\prime} \cdot \mu^{\prime}} .
$$

Then $a_{1}^{-\ell_{1}}, a_{1}^{r_{1}}, a_{2}^{-\ell_{2}}$ and $a_{2}^{r_{2}}$ are nonzero on $\{z \in \mathbb{C}|| z \mid \geq 1\} \times \mathbb{R}^{d-1}$ and have nonzero degree compared with $z$ for all $\left(\eta^{\prime}, \eta_{3-k}\right)$.

Then the result generalizing [Cou15] from the half space to corner spaces is the following:

Theorem 3.2 Under Assumptions 3.3 .3 .4 and 3.5 . Assume that the difference scheme approximation 23. is strongly stable in the sense of Definition 2.1 then (23) is also semi-group stable. More precisely, there exists $C>0$ such that for all $\gamma>0$ and $\Delta t \in] 0,1]$ the solution of 10 satisfies the estimate:

$$
\begin{aligned}
\sup _{n \geq 0} e^{-2 \gamma n \Delta t}\left\|u^{n}\right\|_{\mathscr{R}}^{2} & +\frac{\gamma}{\gamma \Delta t+1} \sum_{n \geq 0} \Delta t e^{-2 \gamma n \Delta t}\left\|u^{n}\right\|_{\mathscr{R}}^{2}+\sum_{k=1}^{2} \sum_{n \geq 0} \Delta t \Delta x_{3-k} e^{-2 \gamma n \Delta t}\left\|u^{n}\right\|_{\mathscr{B}_{k}}^{2} \leq \\
C & \left(\sum_{n=0}^{s}\left\|u_{n}\right\|_{\mathscr{R}}^{2}+\frac{\gamma \Delta t+1}{\gamma} \sum_{n \geq s+1} \Delta t e^{-2 \gamma n \Delta t}\left\|f^{n}\right\|_{\mathscr{I}}^{2}\right. \\
& \left.+\sum_{k=1}^{2} \sum_{n \geq s+1} \Delta t \Delta x_{3-k} e^{-2 \gamma n \Delta t}\left\|g_{k}^{n}\right\|_{\mathscr{B}_{k}}^{2}+\sum_{n \geq s+1} \Delta t \Delta x_{2} e^{-2 \gamma n \Delta t}\left\|h^{n}\right\|_{\mathscr{C}}^{2}\right) .
\end{aligned}
$$

Remark The proof of Theorem 3.1 in fact needs a weaker definition of strong stability. More precisely, Theorem 3.1 remains true if one assumes that the scheme is strongly stable in the sense of Definition 2.1 with $\mathscr{B}_{1}$ and $\mathscr{B}_{2}$ instead of $\overline{\mathscr{B}_{1}}$ and $\overline{\mathscr{B}_{2}}$. However, this is not the case for the proof of Theorem 3.2 .

We now turn to the proofs of Theorems 3.1 and 3.2 . 


\section{Proof of Theorem 3.1}

Following CG11 the finite difference scheme with discrete Dirichlet conditions on each boundary and at the corner will be a suitable (in the sense that we can demonstrate that the semi-group, interior and extended traces norms of its solutions are controlable by the source terms) auxiliary problem.

\subsection{Finite difference schemes with discrete Dirichlet boundary and corner con- ditions}

Let us introduce the auxiliary finite difference scheme of 21) in which we just substitute the boundary and corner conditions by Dirichlet conditions:

$$
\begin{cases}U_{j}^{n+1}+Q U_{j}^{n}=\Delta t f_{j}^{n+1}, & \text { for } j \in \mathscr{I} \times \mathbb{Z}^{d-2}, n \geq 0, \\ U_{j}^{n+1}=g_{1, j}^{n+1}, & \text { for } j \in \mathscr{B}_{1} \times \mathbb{Z}^{d-2}, n \geq 0, \\ U_{j}^{n+1}=g_{2, j}^{n+1}, & \text { for } j \in \mathscr{B}_{2} \times \mathbb{Z}^{d-2}, n \geq 0, \\ U_{j}^{n+1}=h_{j}^{n+1}, & \text { for } j \in \mathscr{C} \times \mathbb{Z}^{d-2}, n \geq 0, \\ U_{j}^{0}=u_{0, j}, & \text { for } j \in \mathscr{R} \times \mathbb{Z}^{d-2} .\end{cases}
$$

The aim of this paragraph is to show that the solution of (25) satisfies the same estimate as in Theorem 3.1 equation (22).

Theorem 4.1 Under Assumptions 3.1 and 3.2, there exists $C>0$ such that for all $\gamma>0$ and $\Delta t \in] 0,1]$ the solution $U$ of 25 satisfies:

$$
\begin{aligned}
\sup _{n \geq 0} e^{-2 \gamma n \Delta t}\left\|U^{n}\right\|_{\mathscr{R}}^{2} & +\frac{\gamma}{\gamma \Delta t+1} \sum_{n \geq 0} \Delta t e^{-2 \gamma n \Delta t}\left\|U^{n}\right\|_{\mathscr{R}}^{2}+\sum_{k=1}^{2} \sum_{n \geq 0} \Delta t \Delta x_{3-k} e^{-2 \gamma n \Delta t}\left\|U^{n}\right\|_{\mathscr{\mathscr { B }}_{k}}^{2} \\
\leq & C\left(\left\|u_{0}\right\|_{\mathscr{R}}^{2}+\frac{\gamma \Delta t+1}{\gamma} \sum_{n \geq 1} \Delta t e^{-2 \gamma n \Delta t}\left\|f^{n}\right\|_{\mathscr{I}}^{2}\right. \\
& \left.+\sum_{k=1}^{2} \sum_{n \geq 1} \Delta t \Delta x_{3-k} e^{-2 \gamma n \Delta t}\left\|g_{k}^{n}\right\|_{\mathscr{B}_{k}}^{2}+\sum_{n \geq 1} \Delta t \Delta x_{2} e^{-2 \gamma n \Delta t}\left\|h^{n}\right\|_{\mathscr{C}}^{2}\right) .
\end{aligned}
$$

The proof of Theorem 4.1 is splitted in two parts. In a first time we show that 26 holds for homogeneous schemes in the interior and in a second time, we show that (26) holds for inhomogeneous schemes in the interior but homogeneous for all the others conditions.

Remark Theorem 4.1 is a key step in the proof of Theorem 3.1. However this result is also interesting for itself. Indeed, it shows that as far as the semi-group stability is concerned, Dirichlet boundary conditions wich are (with Neumann boundary conditions) the simplest ones to use are suitable. This fact is interesting because Dirichlet boundary conditions can lead to severe consistency issues for the finite scheme approximation (21) for example when they are imposed while there exists an outgoing modes (that is a mode which transports the information from the interior of the domain to the boundaries or to the corner).

\subsubsection{Homogeneous schemes in the interior}

In this paragraph we show that the solution of the scheme 25) with homogeneous source term in the interior (that is $f_{j}^{n} \equiv 0$ ) admits a suitable estimate to show that 25) is strongly stable. More precisely we will show the following lemma: 
Lemma 4.1 Under Assumptions 3.1 and 3.2 there exists $C>0$ such that for all $\gamma>0$ and $\Delta t \in] 0,1]$ the solution of 25) (with $f_{j}^{n} \equiv 0$ ) satisfies the estimate:

$$
\begin{aligned}
\sup _{n \geq 0} e^{-2 \gamma n \Delta t}\left\|U^{n}\right\|_{\mathscr{R}}^{2} & +\frac{\gamma}{\gamma \Delta t+1} \sum_{n \geq 0} \Delta t e^{-2 \gamma n \Delta t}\left\|U^{n}\right\|_{\mathscr{R}}^{2}+\sum_{k=1}^{2} \sum_{n \geq 0} \Delta t \Delta x_{3-k} e^{-2 \gamma n \Delta t}\left\|U^{n}\right\|_{\mathscr{B}_{k}}^{2} \\
\leq C & \left(\left\|u_{0}\right\|_{\mathscr{R}}^{2}+\sum_{k=1}^{2} \sum_{n \geq 1} \Delta t \Delta x_{3-k} e^{-2 \gamma n \Delta t}\left\|g_{3-k}^{n}\right\|_{\mathscr{B}_{k}}^{2}+\sum_{n \geq 1} \Delta t \Delta x_{2} e^{-2 \gamma n \Delta t}\left\|h^{n}\right\|_{\mathscr{C}}^{2}\right) .
\end{aligned}
$$

Proof : Following CG11, we introduce $Q:=-(I+\widetilde{Q})$. Note that from the definition of $Q$ (see (11)) we have:

$$
\widetilde{Q}=-\sum_{\mu_{1}=-\ell_{1}}^{r_{1}} \mathbb{A}_{1}^{\mu_{1}} \mathbf{T}_{1}^{\mu_{1}}=-\sum_{\mu_{2}=-\ell_{2}}^{r_{2}} \mathbb{A}_{2}^{\mu_{2}} \mathbf{T}_{2}^{\mu_{2}} .
$$

From Assumption 3.2 we deduce that:

$$
\left\|\widetilde{Q} U^{n}\right\|_{\mathbb{Z}^{2}}^{2}+2\left\langle\widetilde{Q} U^{n}, U^{n}\right\rangle_{\mathbb{Z}^{2}} \leq 0 .
$$

We then introduce $\left(W_{j}^{n}\right)$ the extension of $\left(U_{j}^{n}\right)$ by zero for $j_{1} \leq-\ell_{1}$ or $j_{2} \leq-\ell_{2}$. In particular in view of 28 we have $\widetilde{Q} W_{j}^{n}=\widetilde{Q} U_{j}^{n}$ on $\mathscr{I}$ and $\widetilde{Q} W_{j}^{n}=0$ for $j_{1} \leq-r_{1}-\ell_{1}$ or $j_{2} \leq-r_{2}-\ell_{2}$. We also define the following subsets of $\mathbb{Z}^{2}$ :

$$
\begin{aligned}
\mathscr{E}_{\mathscr{C}} & :=\left\{j \in \mathbb{Z}^{2} \mid j_{1} \in \llbracket 1-\ell_{1}-r_{1},-\ell_{1} \rrbracket, j_{2} \in \llbracket 1-\ell_{2}-r_{2},-\ell_{2} \rrbracket\right\}, \\
\mathscr{E}_{\mathscr{B}_{1}}:= & \left\{j \in \mathbb{Z}^{2} \mid j_{1} \in \llbracket 1-\ell_{1}-r_{1},-\ell_{1} \rrbracket, j_{2} \in \llbracket 1-\ell_{2}, \infty \llbracket\right\}, \\
\mathscr{E}_{\mathscr{B}_{2}}:= & :=\left\{j \in \mathbb{Z}^{2} \mid j_{1} \in \llbracket 1-\ell_{1}, \infty \llbracket, j_{2} \in \llbracket 1-\ell_{2}-r_{2},-\ell_{2} \rrbracket\right\} .
\end{aligned}
$$

Some computations give:

$$
\begin{aligned}
\left\|\widetilde{Q} W^{n}\right\|_{\mathbb{Z}^{2}}^{2} & =\left\|\widetilde{Q} W^{n}\right\|_{j_{1} \geq 1-r_{1}-\ell_{1} \text { and } j_{2} \geq 1-r_{2}-\ell_{2}}^{2} \\
& =\left\|\widetilde{Q} W^{n}\right\|_{\mathscr{E}_{\mathscr{C}}}^{2}+\left\|\widetilde{Q} W^{n}\right\|_{\mathscr{C}}^{2}+\left\|\widetilde{Q} W^{n}\right\|_{\mathscr{E}_{\mathscr{B}_{1}}}^{2}+\left\|\widetilde{Q} W^{n}\right\|_{\mathscr{B}_{1}}^{2}+\left\|\widetilde{Q} W^{n}\right\|_{\mathscr{E}_{\mathscr{B}_{2}}}^{2}+\left\|\widetilde{Q} W^{n}\right\|_{\mathscr{B}_{2}}^{2}+\left\|\widetilde{Q} U^{n}\right\|_{\mathscr{I}}^{2},
\end{aligned}
$$

and

$$
\begin{aligned}
\left\langle\widetilde{Q} W^{n}, W^{n}\right\rangle_{\mathbb{Z}^{2}} & =\left\langle\widetilde{Q} W^{n}, W^{n}\right\rangle_{j_{1} \geq 1-\ell_{1} \text { and } j_{2} \geq 1-\ell_{2}} \\
& =\left\langle\widetilde{Q} W^{n}, W^{n}\right\rangle_{\mathscr{C}}+\left\langle\widetilde{Q} W^{n}, W^{n}\right\rangle_{\mathscr{B}_{1}}+\left\langle\widetilde{Q} W^{n}, W^{n}\right\rangle_{\mathscr{B}_{2}}+\left\langle\widetilde{Q} U^{n}, U^{n}\right\rangle_{\mathscr{I}}
\end{aligned}
$$

Then for $\mathfrak{I} \in\left\{\mathscr{C}, \mathscr{B}_{1}, \mathscr{B}_{2}, \mathscr{I}\right\}$ we write:

$$
\left\|\widetilde{Q} W^{n}\right\|_{\mathfrak{I}}^{2}+2\left\langle\widetilde{Q} W^{n}, W^{n}\right\rangle_{\mathfrak{I}}=\left\|\widetilde{Q} W^{n}+W^{n}\right\|_{\mathfrak{I}}^{2}-\left\|U^{n}\right\|_{\mathfrak{I}}^{2} .
$$

In particular, for $\mathfrak{I}=\mathscr{I}$, using the definition of the scheme 25 , we can simplify the previous expression in the following way:

$$
\left\|\widetilde{Q} U^{n}\right\|_{\mathscr{I}}^{2}+2\left\langle\widetilde{Q} U^{n}, U^{n}\right\rangle_{\mathscr{I}}=\left\|Q U^{n}\right\|_{\mathscr{I}}^{2}-\left\|U^{n}\right\|_{\mathscr{I}}^{2}=\left\|U^{n+1}\right\|_{\mathscr{I}}^{2}-\left\|U^{n}\right\|_{\mathscr{I}}^{2} .
$$

This term will permit to obtain the supremum in time in the estimate of Lemma 4.1 .

From equations $29,, 31,30)$ and the two previous relations we obtain, thanks to the fact that we have Dirichlet boundary and corner conditions, that:

$$
\left\|U^{n+1}\right\|_{\mathscr{I}}^{2}-\left\|U^{n}\right\|_{\mathscr{I}}^{2}+\sum_{\mathfrak{I} \in\left\{\mathscr{\mathscr { C }}, \mathscr{B}_{1}, \mathscr{B}_{2}\right\}}\left\|\widetilde{Q} W^{n}\right\|_{\mathscr{E}_{\mathfrak{I}}}^{2}+\left\|\widetilde{Q} W^{n}+W^{n}\right\|_{\mathfrak{I}}^{2} \leq\left\|g_{1}^{n}\right\|_{\mathscr{B}_{1}}^{2}+\left\|g_{2}^{n}\right\|_{\mathscr{B}_{2}}^{2}+\left\|h^{n}\right\|_{\mathscr{C}^{2}}^{2} .
$$




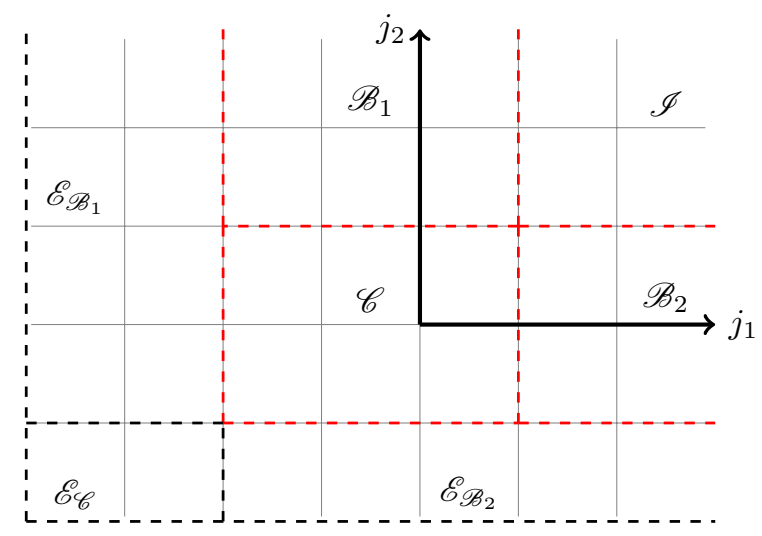

Figure 2: Error terms induced by the extension by zero for $\ell_{1}=3, \ell_{2}=2, r_{1}=2$ and $r_{2}=1$.

In particular we have:

$$
\left\{\begin{array}{c}
\left\|U^{n+1}\right\|_{\mathscr{I}}^{2}-\left\|U^{n}\right\|_{\mathscr{I}}^{2}+\left\|\widetilde{Q} W^{n}\right\|_{\mathscr{E}_{\mathscr{B}_{1}}}^{2}+\left\|\widetilde{Q} W^{n}+W^{n}\right\|_{\mathscr{B}_{1}}^{2}+\left\|\widetilde{Q} W^{n}+W^{n}\right\|_{\mathscr{C}}^{2} \leq\left\|g_{1}^{n}\right\|_{\mathscr{B}_{1}}^{2}+\left\|g_{2}^{n}\right\|_{\mathscr{B}_{2}}^{2}+\left\|h^{n}\right\|_{\mathscr{C}}^{2}, \\
\left\|U^{n+1}\right\|_{\mathscr{I}}^{2}-\left\|U^{n}\right\|_{\mathscr{I}}^{2}+\left\|\widetilde{Q} W^{n}\right\|_{\mathscr{E}_{\mathscr{B}_{2}}}^{2}+\left\|\widetilde{Q} W^{n}+W^{n}\right\|_{\mathscr{B}_{2}}^{2}+\left\|\widetilde{Q} W^{n}+W^{n}\right\|_{\mathscr{C}}^{2} \leq\left\|g_{1}^{n}\right\|_{\mathscr{B}_{1}}^{2}+\left\|g_{2}^{n}\right\|_{\mathscr{B}_{2}}^{2}+\left\|h^{n}\right\|_{\mathscr{C}_{C}}^{2}
\end{array}\right.
$$

The following lemma is a straightforward generalization of [[CG11]- Lemma 2.2] from finite to infinite dimension. It is however the keystone of the analysis.

Lemma 4.2 There exist two constants $c_{1}, c_{2}>0$ such that for all $n \in \mathbb{N}$ we have:

$$
\begin{aligned}
& \left\|\widetilde{Q} W^{n}\right\|_{\mathscr{E}_{\mathscr{B}_{1}}}^{2}+\left\|\widetilde{Q} W^{n}+W^{n}\right\|_{\mathscr{B}_{1}}^{2}+\left\|\widetilde{Q} W^{n}+W^{n}\right\|_{\mathscr{C}}^{2} \geq c_{1}\left\|W^{n}\right\|_{\mathscr{B}_{1}}^{2}, \\
& \left\|\widetilde{Q} W^{n}\right\|_{\mathscr{E}_{\mathscr{B}_{2}}}^{2}+\left\|\widetilde{Q} W^{n}+W^{n}\right\|_{\mathscr{B}_{2}}^{2}+\left\|\widetilde{Q} W^{n}+W^{n}\right\|_{\mathscr{C}}^{2} \geq c_{2}\left\|W^{n}\right\|_{\mathscr{B}_{2}}^{2}
\end{aligned}
$$

The proof of this Lemma is given in paragraph 4.4 .

We apply Lemma 4.2 to 32 , by definition of $\left(W_{j}^{n}\right)$ we obtain:

$$
\left\|U^{n+1}\right\|_{\mathscr{I}}^{2}-\left\|U^{n}\right\|_{\mathscr{I}}^{2}+\left\|U^{n}\right\|_{\mathscr{B}_{1}}^{2}+\left\|U^{n}\right\|_{\mathscr{B}_{2}}^{2} \leq C\left(\left\|g_{1}^{n}\right\|_{\mathscr{B}_{1}}^{2}+\left\|g_{2}^{n}\right\|_{\mathscr{B}_{2}}^{2}+\left\|h^{n}\right\|_{\mathscr{C}}^{2}\right)
$$

we multiply this equation by $\Delta x e^{-2 \gamma n \Delta t}$ and sum for $n \in \llbracket 0, N \rrbracket$. It follows, from the definition of the CFL numbers that:

$$
\begin{aligned}
e^{-2 \gamma N \Delta t}\left\|U^{N+1}\right\|_{\mathscr{I}}^{2} & +\frac{e^{2 \gamma \Delta t}-1}{\Delta t} \sum_{n=1}^{N} \Delta t e^{-2 \gamma n \Delta t}\left\|U^{n}\right\|_{\mathscr{I}}^{2}+\sum_{k=1}^{2} \frac{1}{\lambda_{k}} \sum_{n=0}^{N} \Delta t \Delta x_{3-k}\left\|U^{n}\right\|_{\mathscr{B}_{k}}^{2} \\
& \leq C\left(\left\|u_{0}\right\|_{\mathscr{R}}^{2}+\sum_{k=1}^{2} \frac{1}{\lambda_{k}} \sum_{n \geq 0} \Delta t \Delta x_{3-k} e^{-2 \gamma n \Delta t}\left\|g_{k}^{n}\right\|_{\mathscr{B}_{k}}^{2}+\sum_{n \geq 0} \Delta t \Delta x_{2} e^{-2 \gamma n \Delta t}\left\|h^{n}\right\|_{\mathscr{C}}^{2}\right) .
\end{aligned}
$$

We then take the supremum in $N$ in the previous equation to obtain that for $\Delta t \leq 1$ :

$$
\begin{aligned}
& e^{2 \gamma \Delta t} \sup _{n \geq 1} e^{-2 \gamma n \Delta t}\left\|U^{n}\right\|_{\mathscr{I}}^{2}+\gamma \sum_{n \geq 1} \Delta t e^{-2 \gamma n \Delta t}\left\|U^{n}\right\|_{\mathscr{I}}^{2}+\sum_{k=1}^{2} \sum_{n \geq 0} \Delta t \Delta x_{3-k}\left\|U^{n}\right\|_{\mathscr{B}_{k}}^{2} \\
& \leq C\left(\left\|u_{0}\right\|_{\mathscr{R}}^{2}+\sum_{k=1}^{2} \sum_{n \geq 0} \Delta t \Delta x_{3-k} e^{-2 \gamma n \Delta t}\left\|g_{k}^{n}\right\|_{\mathscr{B}_{k}}^{2}+\sum_{n \geq 0} \Delta t \Delta x_{2} e^{-2 \gamma n \Delta t}\left\|h^{n}\right\|_{\mathscr{C}}^{2}\right) .
\end{aligned}
$$


Then we remark that $\frac{\gamma}{\gamma \Delta t+1} \leq \gamma, \frac{\gamma \Delta t}{\gamma \Delta t+1} \leq 1$ and use the initial condition to obtain:

$$
\begin{aligned}
\sup _{n \geq 0} e^{-2 \gamma n \Delta t}\left\|U^{n}\right\|_{\mathscr{I}}^{2} & +\frac{\gamma}{\gamma \Delta t+1} \sum_{n \geq 0} \Delta t e^{-2 \gamma n \Delta t}\left\|U^{n}\right\|_{\mathscr{I}}^{2}+\sum_{k=1}^{2} \sum_{n \geq 0} \Delta t \Delta x_{3-k}\left\|U^{n}\right\|_{\mathscr{B}_{k}}^{2} \\
\leq & C\left(\left\|u_{0}\right\|_{\mathscr{R}}^{2}+\sum_{k=1}^{2}\left(\Delta t \Delta x_{3-k}\left\|g_{k}^{0}\right\|_{\mathscr{B}_{k}}^{2}+\sum_{n \geq 1} \Delta t \Delta x_{3-k} e^{-2 \gamma n \Delta t}\left\|g_{k}^{n}\right\|_{\mathscr{B}_{k}}^{2}\right)\right. \\
& \left.+\Delta t \Delta x_{2}\left\|h^{0}\right\|_{\mathscr{C}}^{2}+\sum_{n \geq 1} \Delta t \Delta x_{2} e^{-2 \gamma n \Delta t}\left\|h^{n}\right\|_{\mathscr{C}}^{2}\right) .
\end{aligned}
$$

At last using the fact that we have Dirichlet boundary conditions we can bound the right hand side by:

$$
\begin{array}{r}
\sup _{n \geq 0} e^{-2 \gamma n \Delta t} \Delta t\left\|U^{n}\right\|_{\mathscr{I}}^{2}+\frac{\gamma}{\gamma \Delta t+1} \sum_{n \geq 0} \Delta t e^{-2 \gamma n \Delta t}\left\|U^{n}\right\|_{\mathscr{I}}^{2}+\sum_{k=1}^{2} \sum_{n \geq 0} \Delta t \Delta x_{3-k}\left\|U^{n}\right\|_{\mathscr{B}_{k}}^{2} \\
\leq C() .
\end{array}
$$

To conclude the proof of Lemma 4.1 we add in 35 the term:

$$
\sum_{\mathfrak{I} \in\left\{\mathscr{C}, \mathscr{B}_{1}, \mathscr{B}_{2}\right\}} \sup _{n \geq 0} e^{-2 \gamma n \Delta t}\left\|U^{n}\right\|_{\mathfrak{I}}^{2}+\frac{\gamma \Delta t}{\gamma \Delta t+1} \sum_{n \geq 0} e^{-2 \gamma n \Delta t}\left\|U^{n}\right\|_{\mathfrak{I}}^{2}
$$

so that the left hand side of $(33)$ is greater (because $\frac{\gamma \Delta t}{\gamma \Delta t+1}<1$ ) than the left hand side of 27 ). Then we remark, thanks to the fact that we have Dirichlet conditions at the boundaries and the corner, that:

$$
\begin{array}{r}
\left\|U^{n}\right\|_{\mathscr{C}}^{2}=\Delta x_{1} \Delta x_{2} \sum_{j \in \mathscr{C}}\left|U_{j}^{n}\right|^{2}=\frac{1}{\lambda_{1}} \sum_{j \in \mathscr{C}} \Delta t \Delta x_{2}\left|h_{j}^{n}\right|^{2}, \\
\left\|U^{n}\right\|_{\mathscr{B}_{1}}^{2}=\frac{1}{\lambda_{1}} \sum_{j \in \mathscr{C}} \Delta t \Delta x_{2}\left|g_{1, j}^{n}\right|^{2} \text {, and }\left\|U^{n}\right\|_{\mathscr{B}_{2}}^{2}=\frac{1}{\lambda_{2}} \sum_{j \in \mathscr{C}} \Delta t \Delta x_{1}\left|g_{2, j}^{n}\right|^{2},
\end{array}
$$

which are exactly the weighted norms of the source terms appearing in the right hand side of (27). We then conclude by using the injection $\ell^{1} \subset \ell^{\infty}$.

\subsubsection{Homogeneous Dirichlet conditions}

We now turn to schemes which only have a nonzero source term in the interior. The result is the following:

Lemma 4.3 Under Assumptions 3.1 and 3.2 there exists $C>0$ such that for all $\gamma>0$ and $\Delta t \in] 0,1]$ the solution of (10) (with $g_{1}^{n}, g_{2}^{n}, h^{n}, u_{0} \equiv 0$ ) satisfies the estimate:

$$
\begin{array}{r}
\sup _{n \geq 0} e^{-2 \gamma n \Delta t}\left\|U_{j}^{n}\right\|_{\mathscr{R}}^{2}+\frac{\gamma}{\gamma \Delta t+1} \sum_{n \geq 0} \Delta t e^{-2 \gamma n \Delta t}\left\|U^{n}\right\|_{\mathscr{R}}^{2}+\sum_{k=1}^{2} \sum_{n \geq 0} \Delta t \Delta x_{3-k} e^{-2 \gamma n \Delta t}\left\|U_{j}^{n}\right\|_{\mathscr{B}_{k}}^{2} \\
\leq C \frac{\gamma \Delta t+1}{\gamma} \sum_{n \geq 1} \Delta t e^{-2 \gamma n \Delta t}\left\|f^{n}\right\|_{\mathscr{I}}^{2}
\end{array}
$$

Proof : First we compute:

$$
\begin{aligned}
\left\|U^{n+1}\right\|_{\mathscr{I}}^{2}-\left\|U^{n}\right\|_{\mathscr{I}}^{2} & :=\left\|(I+\widetilde{Q}) U^{n}+\Delta t f^{n}\right\|_{\mathscr{I}}^{2}-\left\|U^{n}\right\|_{\mathscr{I}}^{2} \\
& =\left\|\widetilde{Q} U^{n}\right\|_{\mathscr{I}}^{2}+2\left\langle\widetilde{Q} U^{n}, U^{n}\right\rangle_{\mathscr{I}}+2 \Delta t\left\langle Q U^{n}, f^{n}\right\rangle_{\mathscr{I}}+\Delta t^{2}\left\|f^{n}\right\|_{\mathscr{I}}^{2}
\end{aligned}
$$


Proceeding as in the proof of Lemma 4.1, we define $\left(W_{j}^{n}\right)$ the extension of $\left(U_{j}^{n}\right)$ by zero for $j_{1} \leq-\ell_{1}$ or $j_{2} \leq-\ell_{2}$. If $r_{1}$ and $r_{2}$ are nonzero then 4.2 holds and we can repeat exactly the same computations as those made in the proof of Lemma 4.1. This leads us to the following inequality (where we strongly used the fact that the conditions at the boundaries and at the corner are homogeneous Dirichlet):

$$
\left\|U^{n+1}\right\|_{\mathscr{I}}^{2}-\left\|U^{n}\right\|_{\mathscr{I}}^{2}+\left\|U^{n}\right\|_{\mathscr{B}_{1}}^{2}+\left\|U^{n}\right\|_{\mathscr{B}_{2}}^{2} \leq C\left(\Delta t\left\langle Q U^{n}, f^{n}\right\rangle_{\mathscr{I}}+\Delta t^{2}\left\|f^{n}\right\|_{\mathscr{I}}^{2}\right) .
$$

As in the proof of Lemma 4.1. we multiply the previous inequality by $\Delta x e^{-2 \gamma n \Delta t}$ and sum over $n \in \llbracket 0, N \rrbracket$. This gives from the definition of the CFL numbers and by Cauchy-Schwartz inequality:

$$
\begin{aligned}
e^{-2 \gamma N \Delta t}\left\|U^{N+1}\right\|_{\mathscr{I}}^{2} & +\frac{e^{2 \gamma \Delta t}-1}{\Delta t} \sum_{n=1}^{N} \Delta t e^{-2 \gamma n \Delta t}\left\|U^{n}\right\|_{\mathscr{I}}^{2}+\sum_{k=1}^{2} \sum_{n=0}^{N} \Delta t \Delta x_{3-k} e^{-2 \gamma n \Delta t}\left\|U^{n}\right\|_{\mathscr{B}_{k}}^{2} \\
& \leq C\left(\Delta t \sum_{n=0}^{N} \Delta t e^{-2 \gamma n \Delta t}\left\|f^{n}\right\|_{\mathscr{I}}^{2}+\sum_{n=0}^{N} e^{-2 \gamma n \Delta t} \Delta t\left\|\sqrt{\Delta x} U^{n}\right\| \mathscr{I}\left\|\sqrt{\Delta x} f^{n}\right\|_{\mathscr{I}}\right) .
\end{aligned}
$$

To conclude we use Young's inequality (with parameter $\frac{e^{2 \gamma \Delta t}-1}{2 \Delta t}$ ) in the last term of the right hand side and it follows that:

$$
\begin{aligned}
e^{-2 \gamma N \Delta t}\left\|U^{N+1}\right\|_{\mathscr{I}}^{2} & +\frac{e^{2 \gamma \Delta t}-1}{2 \Delta t} \sum_{n=1}^{N} \Delta t e^{-2 \gamma n \Delta t}\left\|U^{n}\right\|_{\mathscr{I}}^{2}+\sum_{k=1}^{2} \sum_{n=0}^{N} \Delta t \Delta x_{3-k} e^{-2 \gamma n \Delta t}\left\|U^{n}\right\|_{\mathscr{B}_{k}}^{2} \\
& \leq C \frac{2 \Delta t}{e^{2 \gamma \Delta t}+1} \sum_{n=0}^{N} e^{-2 \gamma(n+1) \Delta t} \Delta t\left\|f^{n}\right\|_{\mathscr{I}}^{2} .
\end{aligned}
$$

The result is obtained from the inequality $\frac{e^{2 \gamma \Delta t}-1}{2 \Delta t} \leq \gamma \leq \frac{\gamma}{\gamma \Delta t+1}$ and by taking the supremum in $N$ (recall once again that the initial condition is zero).

\subsection{Reinforcement of traces estimates}

The solution of the auxiliary problem (25), $\left(U_{j}^{n}\right)$, and more especifically its traces will act like an error term in the final error analysis in the proof of Theorem 3.1. More precisely we will have to control the terms $B_{1} U_{i, j_{2}, j^{\prime}}, B_{2} U_{j_{1}, 1, j^{\prime}}$ and finally $C U_{i, 1, j^{\prime}}$ which may involve more terms than those controled by 26]. It is typically the case if some of the parameters (for example $q_{11}$ or $q_{22}$ ) are larger than $r_{1}$ and $r_{2}$. As a consequence to perform the final error analysis, we need to obtain a better control of the traces in (26). The result is the following:

Theorem 4.2 Under Assumptions 3.1 and 3.2, let $P_{1}, P_{2}$ be two fixed integers, then there exists $C>0$ such that for all $\gamma>0$ the solution $\left(U_{j}^{n}\right)$ of 25 satisfies:

$$
\begin{aligned}
\sup _{n \geq 0} e^{-2 \gamma n \Delta t}\left\|U^{n}\right\|_{\mathscr{R}}^{2} & +\frac{\gamma}{\gamma \Delta t+1} \sum_{n \geq 0} \Delta t e^{-2 \gamma n \Delta t}\left\|U^{n}\right\|_{\mathscr{R}}^{2}+\sum_{k=1}^{2} \sum_{n \geq 0} \Delta t \Delta x_{3-k} e^{-2 \gamma n \Delta t} \sum_{j_{k}=1-\ell_{k}}^{P_{k}}\left\|U_{j_{k},}^{n},\right\|_{\ell^{2}(\mathbb{Z} \alpha 36)}^{2}\left(\left\|u_{0}\right\|_{\mathscr{R}}^{2}+\frac{\gamma \Delta t+1}{\gamma} \sum_{n \geq 1} \Delta t e^{-2 \gamma(n+1) \Delta t}\left\|f^{n}\right\|_{\mathscr{I}}^{2}\right. \\
\leq & C \\
& \left.+\sum_{k=1}^{2} \sum_{n \geq 1} \Delta t \Delta x_{3-k} e^{-2 \gamma n \Delta t}\left\|g_{k}^{n}\right\|_{\mathscr{B}_{k}}^{2}+\sum_{n \geq 1} \Delta t \Delta x_{2} e^{-2 \gamma n \Delta t}\left\|h^{n}\right\|_{\mathscr{C}}^{2}\right)
\end{aligned}
$$

The proof follows, once again, the proof given in CG11] up to some little difficulties induced by the corner geometry. 
Proof : Let us define the shifted sequence $V_{j}^{n}:=U_{j_{1}+1, j_{2}, j^{\prime}}^{n}$. Then, for all $n \geq 0,\left(V_{j}^{n}\right)$ solves the equation:

$$
\begin{cases}V_{j}^{n+1}+Q V_{j}^{n}=\Delta t f_{j_{1}+1, j_{2}, j^{\prime}}^{n+1} & , j \in \mathscr{I}, \\ V_{j}^{n+1}=g_{2, j_{1}+1, j_{2}, j^{\prime}}^{n+1} & , j_{1} \in \llbracket 1, \infty \llbracket \text { and }\left(j_{2}, j^{\prime}\right) \in \llbracket 1-\ell_{2}, 0 \rrbracket \times \mathbb{Z}^{d-2}, \\ V_{j}^{n+1}=g_{1, j_{1}+1, j_{2}, j^{\prime}}^{n+1} & , j_{1} \in \llbracket 1-\ell_{1},-1 \rrbracket \text { and }\left(j_{2}, j^{\prime}\right) \in \llbracket 1, \infty \llbracket \times \mathbb{Z}^{d-2}, \\ V_{j}^{n+1}=h_{1, j_{1}+1, j_{2}, j^{\prime}}^{n+1} & , j_{1} \in \llbracket 1-\ell_{1},-1 \rrbracket \text { and }\left(j_{2}, j^{\prime}\right) \in \llbracket 1-\ell_{2}, 0 \rrbracket \times \mathbb{Z}^{d-2}, \\ V_{j}^{n+1}=U_{1, j_{2}, j^{\prime}}^{n+1} & , j_{1}=0 \text { and }\left(j_{2}, j^{\prime}\right) \in \llbracket 1, \infty \llbracket \times \mathbb{Z}^{d-2}, \\ V_{j}^{n+1}=g_{2,1, j_{2}, j^{\prime}}^{n+1} & , j_{1}=0 \text { and }\left(j_{2}, j^{\prime}\right) \in \llbracket 1-\ell_{2}, 0 \rrbracket \times \mathbb{Z}^{d-2}, \\ V_{j}^{0}=u_{0, j_{1}+1, j_{2}, j^{\prime}} & , j \in \mathscr{R} .\end{cases}
$$

So we can apply Theorem 4.1 to $\left(V_{j}^{n}\right)$ to obtain the estimat 4

$$
\begin{aligned}
\Delta t \Delta x_{2} e^{-2 \gamma n \Delta t}\left\|V_{r_{1}, \cdot}^{n}\right\|_{\ell_{j_{2}, j^{\prime}}^{2}\left(\mathbb{Z}^{d-2}\right)}^{2} \leq & C\left(\left\|u_{0}\right\|_{\mathscr{R}}^{2}+\frac{\gamma \Delta t+1}{\gamma} \sum_{n \geq 1} \Delta t e^{-2 \gamma(n+1) \Delta t}\left\|f^{n}\right\|_{\mathscr{I}}^{2}\right. \\
& +\sum_{k=1}^{2} \sum_{n \geq 1} \Delta t \Delta x_{3-k} e^{-2 \gamma n \Delta t}\left\|g_{k}^{n}\right\|_{\mathscr{B}_{k}}^{2} \\
& \left.+\sum_{n \geq 1} \Delta t \Delta x_{2} e^{-2 \gamma n \Delta t}\left\|h^{n}\right\|_{\mathscr{C}}^{2}+\sum_{n \geq 1} \Delta t \Delta x_{2} \sum_{j_{2}=1}^{\infty}\left\|U_{1, j_{2},}^{n},\right\|_{\ell^{2}\left(\mathbb{Z}^{d-3}\right.}^{2}\right) .
\end{aligned}
$$

We then apply again Theorem 4.1 to estimate the last term in the right hand side of (37) (this is effectively possible because $r_{1} \geq 1$ ) and we obtain the following control of $U_{r_{1}+1,}^{n}$ :

$$
\begin{aligned}
\left.\Delta t \Delta x_{2} e^{-2 \gamma n \Delta t} \| U_{r_{1}+1, \|_{\ell_{2}, j^{\prime}}^{n}}^{n} \mathbb{Z}^{d-2}\right) \leq & C\left(\left\|u_{0}\right\|_{\mathscr{R}}^{2}+\frac{\gamma \Delta t+1}{\gamma} \sum_{n \geq 1} \Delta t e^{-2 \gamma(n+1) \Delta t}\left\|f^{n}\right\|_{\mathscr{I}}^{2}\right. \\
& \left.+\sum_{k=1}^{2} \sum_{n \geq 1} \Delta t \Delta x_{3-k} e^{-2 \gamma n \Delta t}\left\|g_{k}^{n}\right\|_{\mathscr{B}_{k}}^{2}+\sum_{n \geq 1} \Delta t \Delta x_{2} e^{-2 \gamma n \Delta t}\left\|h^{n}\right\|_{\mathscr{C}}^{2}\right) .
\end{aligned}
$$

We can then repeat exactly the same reasoning to the sequence $\widetilde{V}_{j}^{n}:=V_{j_{1}+1, j_{2}, j^{\prime}}$ to obtain the analogous of (39) but for $U_{r_{1}+2, .}^{n}$. By induction we can then show that for all $P_{1} \geq r_{1}+1$ (note that the case $P_{1} \leq r_{1}$ is already included in Theorem 4.1. We thus obtain that:

$$
\begin{aligned}
\Delta t \Delta x_{2} e^{-2 \gamma n \Delta t} \sum_{j_{1}=r_{1}+1}^{P_{1}}\left\|U_{j_{1},}^{n},\right\|_{\ell_{2}^{2}, j^{\prime}}^{2}\left(\mathbb{Z}^{d-2}\right) \leq & C\left(\left\|u_{0}\right\|_{\mathscr{R}}^{2}+\frac{\gamma \Delta t+1}{\gamma} \sum_{n \geq 1} \Delta t e^{-2 \gamma(n+1) \Delta t}\left\|f^{n}\right\|_{\mathscr{I}}^{2}\right. \\
& \left.+\sum_{k=1}^{2} \sum_{n \geq 1} \Delta t \Delta x_{3-k} e^{-2 \gamma n \Delta t}\left\|g_{k}^{n}\right\|_{\mathscr{B}_{k}}^{2}+\sum_{n \geq 1} \Delta t \Delta x_{2} e^{-2 \gamma n \Delta t}\left\|h^{n}\right\|_{\mathscr{C}}^{2}\right),
\end{aligned}
$$

which combined with Theorem 4.1 give the desired version of $(36)$ for $P_{2} \leq r_{2}$.

To obtain (36) for arbitrary $P_{2}$ it is sufficient to reiterate the same arguments but with the shifted sequence $W_{j}^{n}:=U_{j_{1}, j_{2}+1, j^{\prime}}^{n}$ and this completes the proof of Theorem 4.2 .

\footnotetext{
${ }^{4}$ Note that the term in the right hand side is not sharp because of the shift but it will be sufficient for our discussion.
} 


\subsection{End of the proof by error estimate}

With Theorem 4.2 in hand the proof of Theorem 3.1 is just an error analysis. More precisely, let $\left(U_{j}^{n}\right)$ be the solution of $\sqrt{10}$, we decompose $U_{j}^{n}:=V_{j}^{n}+W_{j}^{n}$ where $\left(V_{j}^{n}\right)$ and $\left(W_{j}^{n}\right)$ solve respectively:

$$
\begin{cases}V_{j}^{n+1}+Q V_{j}^{n}=\Delta t f_{j}^{n+1} & , \text { for } n \geq 0, j \in \mathscr{I} \times \mathbb{Z}^{d-2} \\ V_{j}^{n+1}=g_{1, j}^{n+1} & , \text { for } n \geq 0, j \in \mathscr{B}_{1} \times \mathbb{Z}^{d-2} \\ V_{j}^{n+1}=g_{2, j}^{n+1} & , \text { for } n \geq 0, j \in \mathscr{B}_{2} \times \mathbb{Z}^{d-2} \\ V_{j}^{n+1}=h_{j}^{n+1} & , \text { for } n \geq 0, j \in \mathscr{C} \times \mathbb{Z}^{d-2} \\ V_{j}^{0}=u_{0, j} & , \text { for } j \in \mathscr{R} \times \mathbb{Z}^{d-2}\end{cases}
$$

and

$$
\begin{cases}W_{j}^{n+1}+Q W_{j}^{n}=0 & , \text { for } n \geq 0, j \in \mathscr{I} \times \mathbb{Z}^{d-2}, \\ W_{j}^{n+1}+\sum_{\sigma=0}^{1} B_{1}^{\sigma, j_{1}} W_{1, j_{2}}^{n+\sigma}=\widetilde{g}_{1, j}^{n+1} & , \text { for } n \geq 0, j \in \mathscr{B}_{1} \times \mathbb{Z}^{d-2}, \\ W_{j}^{n+1}+\sum_{\sigma=0}^{1} B_{2}^{\sigma, j_{2}} W_{j_{1}, 1}^{n+\sigma}=\widetilde{g}_{2, j}^{n+1} & , \text { for } n \geq 0, j \in \mathscr{B}_{2} \times \mathbb{Z}^{d-2}, \\ W_{j}^{n+1}+\sum_{\sigma=0}^{1} C^{\sigma, j_{1}, j_{2}} W_{1,1}^{n+\sigma}=\widetilde{h}_{j}^{n+1} & , \text { for } n \geq 0, j \in \mathscr{C} \times \mathbb{Z}^{d-2}, \\ W_{j}^{0}=0 & , \text { for } j \in \mathscr{R} \times \mathbb{Z}^{d-2},\end{cases}
$$

where the sequences $\widetilde{g}_{1}, \widetilde{g}_{2}$ and $\widetilde{h}$ are defined by: for all $n \leq 0$,

$$
\begin{array}{r}
\forall j \in \mathscr{B}_{1} \times \mathbb{Z}^{d-2}, \widetilde{g}_{1, j}^{n}:=-\sum_{\sigma=0}^{1} B_{1}^{\sigma, j_{1}} V_{1, j_{2}, j^{\prime}}^{n+\sigma}, \\
\forall j \in \mathscr{B}_{2} \times \mathbb{Z}^{d-2}, \widetilde{g}_{2, j}^{n}:=-\sum_{\sigma=0}^{1} B_{2}^{\sigma, j_{2}} V_{j_{1}, 1, j^{\prime}}^{n+\sigma}, \\
\text { and } \forall j \in \mathscr{C} \times \mathbb{Z}^{d-2}, \widetilde{h}_{j}^{n}:=-\sum_{\sigma=0}^{1} C^{\sigma, j_{1}, j_{2}} V_{1,1, j^{\prime}}^{n+\sigma}
\end{array}
$$

By construction, $\left(V_{j}^{n}\right)$ satisfies the estimate (36) so we only have to estimate $\left(W_{j}^{n}\right)$. We use the fact that (21) is assumed to be strongly stable is the sense of Definition 2.1 to obtain the estimate:

$$
\begin{aligned}
\frac{\gamma}{\gamma \Delta t+1} \sum_{n \geq 0} \Delta t e^{-2 \gamma n \Delta t}\left\|W^{n}\right\|_{\mathscr{R}}^{2}+\sum_{k=1}^{2} \sum_{n \geq 0} \Delta t \Delta x_{3-k} e^{-2 \gamma n \Delta t}\left\|W^{n}\right\|_{\mathscr{\mathscr { B }}_{k}}^{2} \leq \\
C\left(\sum_{k=1}^{2} \sum_{n \geq 1} \Delta t \Delta x_{3-k} e^{-2 \gamma n \Delta t}\left\|\widetilde{g}_{k}^{n}\right\|_{\mathscr{B}_{k}}^{2}+\sum_{n \geq 1} \Delta t \Delta x_{2} e^{-2 \gamma n \Delta t}\left\|\widetilde{h}^{n}\right\|_{\mathscr{C}}^{2}\right) .
\end{aligned}
$$

We now turn to the estimate of the right hand side of 441 , thanks to the reinforced traces estimate for $\left(V_{j}^{n}\right)$, that is to say (36). From (40) we have to estimate $\left|B_{1}^{\sigma, j_{1}} V_{1, j_{2}}^{n+\sigma}\right|^{2},\left|B_{2}^{\sigma, j_{2}} V_{j_{1}, 1}^{n+\sigma}\right|^{2}$ and $\left|C^{\sigma, j_{1}, j_{2}} V_{1,1}^{n+\sigma}\right|^{2}$ and we have to distinguish three cases depending of the definition of the operators $B_{1}^{\sigma, j_{1}}, B_{2}^{\sigma, j_{2}}$ and $C^{\sigma, j_{1}, j_{2}}$ (see paragraph 2.2):

$\diamond 10$ admits decoupled boundary and corner conditions:

In this framework, independently of $\sigma$, we have that:

- for $j \in \mathscr{B}_{1}, B_{1}^{\sigma, j_{1}} V_{1, j_{2}}^{n+\sigma}$ involves the $\left|V_{\tilde{j}}^{n}\right|^{2}$ for $\widetilde{j}_{1} \in \llbracket 1,1+q_{11} \rrbracket, \widetilde{j}_{2} \in \llbracket j_{2}, j_{2}+q_{12} \rrbracket$; for $j \in \mathscr{B}_{2}, B_{1}^{\sigma, j_{2}} V_{j_{1}, 1}^{n+\sigma}$ involves the $\left|V_{\tilde{j}}^{n}\right|^{2}$ for $\widetilde{j}_{1} \in \llbracket j_{1}, j_{1}+q_{21} \rrbracket, \widetilde{j}_{2} \in \llbracket 1,1+q_{22} \rrbracket$;

- finally for $j \in \mathscr{C}, C^{\sigma, j_{1}, j_{2}} V_{1,1}^{n+\sigma}$ involves the $\left|V_{\tilde{j}}^{n}\right|^{2}$ for $\widetilde{j}_{1} \in \llbracket 1,1+c_{1} \rrbracket, \widetilde{j}_{2} \in \llbracket 1,1+c_{2} \rrbracket$.

Consequently: 
- the error term $\left\|\widetilde{g}_{1}^{n}\right\|_{\mathscr{B}_{1}}^{2}$ involves the $\left|V_{j}^{n}\right|^{2}$ for $j_{1} \in \llbracket 1,1+q_{11} \rrbracket, j_{2} \geq 1$;

the error term $\left\|\widetilde{g}_{2}^{n}\right\|_{\mathscr{B}_{2}}^{2}$ involves the $\left|V_{j}^{n}\right|^{2}$ for $j_{1} \geq 1, j_{2} \in \llbracket 1,1+q_{22} \rrbracket$;

- and finally the error term $\left\|\widetilde{h}_{1}^{n}\right\|_{\mathscr{C}}^{2}$ involves the $\left|V_{i}^{n}\right|^{2}$ for $j_{1} \in \llbracket 1,1+c_{1} \rrbracket, j_{2} \in \llbracket 1,1+c_{2} \rrbracket$.

As first noticed in [CG11] if $r_{1} \geq q_{11}$ then we use [26), while if $r_{1} \leq q_{11}+1$ we use (36) with $P_{1}=q_{11}+1$. In both cases we obtain:

$$
\begin{aligned}
\sum_{n \geq 0} \Delta t \Delta x_{2} e^{-2 \gamma n \Delta t}\left\|\widetilde{g}_{1}^{n}\right\|_{\mathscr{B}_{1}}^{2} \leq C\left(\left\|u_{0}\right\|_{\mathscr{R}}^{2}\right. & +\frac{\gamma \Delta t+1}{\gamma} \sum_{n \geq 1} \Delta t e^{-2 \gamma(n+1) \Delta t}\left\|f^{n}\right\|_{\mathscr{I}}^{2} \\
& \left.+\sum_{k=1}^{2} \sum_{n \geq 1} \Delta t \Delta x_{3-k} e^{-2 \gamma n \Delta t}\left\|g_{k}^{n}\right\|_{\mathscr{B}_{k}}^{2}+\sum_{n \geq 1} \Delta t \Delta x_{2} e^{-2 \gamma n \Delta t}\left\|h^{n}\right\|_{\mathscr{C}}^{2}\right) .
\end{aligned}
$$

The estimate for the term depending on $\left\|\widetilde{g}_{2}^{n}\right\|_{\mathscr{B}_{2}}^{2}$ in the right hand side of (41) follows exactly the same discussion upon $r_{2}$ and $q_{22}$. This permits to show that $\sum_{n \geq 0} \Delta t \Delta x_{1} e^{-2 \gamma n \Delta t}\left\|\widetilde{g}_{2}^{n}\right\|_{\mathscr{B}_{2}}^{2}$ is bounded by the right hand side of 42 .

Finally to estimate the term depending on $\left\|\widetilde{h}^{n}\right\|_{\mathscr{C}}^{2}$ in the right hand side of 41) we use the fact that in (41), $\left\|\widetilde{h}^{n}\right\|_{\mathscr{C}}^{2}$ and $\left\|\widetilde{g}_{1}^{n}\right\|_{\mathscr{B}_{1}}^{2}$ have the same weight in terms of $\Delta t$ and $\Delta x_{2}$. As a consequence (26) gives the desired bound (that is the right hand side of (42)) if $r_{1} \geq c_{1}$, while if $r_{1} \leq c_{1}+1$ we use (36) with $P_{1}=c_{1}+1$.

We thus have shown that:

$$
\begin{aligned}
\frac{\gamma}{\gamma \Delta t+1} \sum_{n \geq 0} \Delta t e^{-2 \gamma n \Delta t}\left\|W^{n}\right\|_{\mathscr{R}}^{2} & +\sum_{k=1}^{2} \sum_{n \geq 0} \Delta t \Delta x_{3-k} e^{-2 \gamma n \Delta t}\left\|W^{n}\right\|_{\mathscr{\mathscr { B }}_{k}}^{2} \leq \\
& C\left(\left\|u_{0}\right\|_{\mathscr{I}}^{2}+\frac{\gamma \Delta t+1}{\gamma} \sum_{n \geq 1} \Delta t e^{-2 \gamma(n+1) \Delta t}\left\|f^{n}\right\|_{\mathscr{I}}^{2}\right. \\
& \left.+\sum_{k=1}^{2} \sum_{n \geq 1} \Delta t \Delta x_{3-k} e^{-2 \gamma n \Delta t}\left\|g_{k}^{n}\right\|_{\mathscr{B}_{k}}^{2}+\sum_{n \geq 1} \Delta t \Delta x_{2} e^{-2 \gamma n \Delta t}\left\|h^{n}\right\|_{\mathscr{C}}^{2}\right) .
\end{aligned}
$$

$\diamond(10)$ is of type traces to corner: From Definition 2.2 , the terms depending on $\left\|\widetilde{g}_{k}^{n}\right\|_{\mathscr{B}_{k}}^{2}$ in the right hand side of (41) are bounded as in the previous case.

In view of the definition of $C^{\sigma, j_{1}, j_{2}}$, the norm $\left\|\widetilde{h}_{1}^{n}\right\|_{\mathscr{C}}^{2}$ involves the $\left|V_{j}^{n}\right|^{2}$ for

$$
\left(j_{1}, j_{2}\right) \in\left(\llbracket 1,1+c_{1} \rrbracket \times \llbracket 1,1+c_{2} \rrbracket\right) \cup\left(\llbracket 1,1+c_{11} \rrbracket \times \llbracket 1-\ell_{2}, 0 \rrbracket\right) \cup\left(\llbracket 1-\ell_{1}, 0 \rrbracket \times \llbracket 1,1+c_{22} \rrbracket\right) .
$$

To estimate the terms $\left|V_{j}^{n}\right|^{2}$ for $\left(j_{1}, j_{2}\right) \in \llbracket 1,1+c_{1} \rrbracket \times \llbracket 1,1+c_{2} \rrbracket$ (resp. $\left.\llbracket 1,1+c_{11} \rrbracket \times \llbracket 1-\ell_{2}, 0 \rrbracket\right)$ we use (26) or (36) applied to $P_{1}=1+c_{1}$ (resp. $\left.P_{1}=1+c_{11}\right)$ depending of the sign of $r_{1}-c_{1}$ (resp. $\left.r_{1}-c_{11}\right)$. Finally to estimate $\left|V_{i}^{n}\right|^{2}$ for $\left(j_{1}, j_{2}\right) \in \llbracket 1-\ell_{1}, 0 \rrbracket \times \llbracket 1,1+c_{22} \rrbracket$ we remark that CFL condition $\frac{x_{2}}{\lambda_{1}}=\frac{x_{1}}{\lambda_{2}}$ and we then use 26) or (36) applied to $P_{2}=1+c_{22}$, estimate 43 follows.

$\diamond 100$ is of type corner to traces: In the case, the proof of the estimate 43 follows exactly the same arguments as when (10) admits decoupled boundary and corner conditions so it will not be treated here.

In view of 10 to conclude the proof of Theorem 3.1 we just have to show that $\sup _{n \leq 0} e^{-2 \gamma n \Delta t}\left\|W^{n}\right\| \|_{\mathscr{R}}^{2}$ can be bounded by the left hand side of 433 . The proof follows exactly the same arguments as in [CG11], paragraph 2.3 and Appendix A] so we will not give the details here.

\subsection{Proof of Lemma 4.2}

We will only show here the first equality in Lemma 4.2 , the proof of the second one is totally equivalent. Let us rewrite the first equality in Lemma 4.2 as:

$$
\sum_{j_{1}=1-r_{1}-\ell_{1}}^{-r_{1}}\left\|\widetilde{Q} W_{j_{1}}^{n},\right\|_{\ell^{2}\left(\mathbb{Z}^{d-1}\right)}^{2}+\sum_{j_{1}=1-\ell_{1}}^{0}\left\|\left(\widetilde{Q} W^{n}+W^{n}\right)_{j_{1},} \cdot\right\|_{\ell^{2}\left(\mathbb{Z}^{d-1}\right)}^{2} \geq c \sum_{j_{1}=1-\ell_{1}}^{r_{1}}\left\|W_{j_{1},}^{n} \cdot\right\|_{\ell^{2}\left(\mathbb{Z}^{d-1}\right)}^{2} .
$$


In view of the definitions of $\widetilde{Q}$ and $\left(W_{j}^{n}\right)$, we have that:

$$
\begin{aligned}
\sum_{j_{1}=1-r_{1}-\ell_{1}}^{-r_{1}}\left\|\widetilde{Q} W_{j_{1}, \cdot}^{n}\right\|_{\ell^{2}\left(\mathbb{Z}^{d-1}\right)}^{2} & =\sum_{j_{1}=1-r_{1}-\ell_{1}}^{-r_{1}}\left\|\mathbb{A}_{1}^{r_{1}} W_{j_{1}+r_{1}, \cdot}^{n}+\sum_{k<r_{1}} \mathbb{A}^{k} W_{j_{1}+k, \cdot}^{n}\right\|_{\ell^{2}\left(\mathbb{Z}^{d-1}\right)}^{2}, \\
& =\left\|\mathbb{A}_{1}^{r_{1}} W_{1-\ell_{1}}^{n},\right\|_{\ell^{2}\left(\mathbb{Z}^{d-1}\right)}^{2}+\sum_{j_{1}=2-r_{1}-\ell_{1}}^{-r_{1}}\left\|\mathbb{A}_{1}^{r_{1}} W_{1-\ell_{1}, \cdot}^{n}+\sum_{k<r_{1}} \mathbb{A}_{1}^{k} W_{j_{1}+k, \cdot}^{n}\right\|_{\ell^{2}\left(\mathbb{Z}^{d-1}\right)}^{2} .
\end{aligned}
$$

As a consequence, equation (44) can be rewritten under the form:

$$
\begin{aligned}
\left\|\mathscr{L} W_{1-\ell_{1},}^{n} \cdot\right\|_{\ell^{2}\left(\mathbb{Z}^{d-1}\right)}^{2}+\left\|\mathscr{L} W_{2-\ell_{1}, \cdot}^{n}+\mathscr{L}_{1} W_{1-\ell_{1}, .}^{n}\right\|^{2}+\ldots+\left\|\mathscr{L} W_{r_{1}, \cdot}^{n}+\mathscr{L}_{\ell_{1}+r_{1}-1}\left(W_{1-\ell_{1}, \cdot}^{n}, \ldots, W_{r_{1}-1, \cdot}^{n}\right)\right\|^{2} \\
\geq \sum_{j_{1}=1-\ell_{1}}^{r_{1}}\left\|W_{j_{1}, .}^{n}\right\|_{\ell^{2}\left(\mathbb{Z}^{d-1}\right)}^{2},
\end{aligned}
$$

where $\mathscr{L}:=\mathbb{A}_{1}^{r_{1}}$ and where the $\mathscr{L}_{k}$ are linear and bounded on $\ell^{2}\left(\mathbb{Z}^{d-1}\right)^{k}$ (the precise expression of these operators is not usefull for what follows).

Then we proceed by indution on $r_{1}$ and contradiction. Firstly we assume that for all $k \in \mathbb{N}$, there exist two sequences $X_{1}^{k}, X_{2}^{k} \in \ell^{2}\left(\mathbb{Z}^{d-1}\right)$ such that $\left\|X_{1}^{k}\right\|_{\ell^{2}\left(\mathbb{Z}^{d-1}\right)}^{2}+\left\|X_{2}^{k}\right\|_{\ell^{2}\left(\mathbb{Z}^{d-1}\right)}^{2}=1$ and satisfying:

$$
\forall k \in \mathbb{N}, \quad\left\|\mathscr{L} X_{1}^{k}\right\|_{\ell^{2}\left(\mathbb{Z}^{d-1}\right)}^{2}+\left\|\mathscr{L} X_{2}^{k}+\mathscr{L}_{1} X_{1}^{k}\right\|_{\ell^{2}\left(\mathbb{Z}^{d-1}\right)}^{2} \leq \frac{1}{k} .
$$

Thus we have that $\left\|\mathscr{L} X_{1}^{k}\right\|_{\ell^{2}\left(\mathbb{Z}^{d-1}\right)} \downarrow 0$ which implies, by Assumption 3.1. that $\left\|X_{1}^{k}\right\|_{\ell^{2}\left(\mathbb{Z}^{d-1}\right) \downarrow 0 \text { and thus }}$

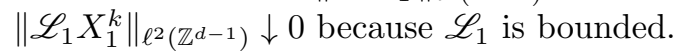

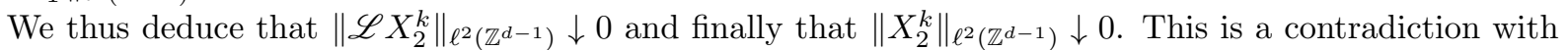
the fact that $\left\|X_{1}^{k}\right\|_{\ell^{2}\left(\mathbb{Z}^{d-1}\right)}^{2}+\left\|X_{2}^{k}\right\|_{\ell^{2}\left(\mathbb{Z}^{d-1}\right)}^{2}=1$. So (44) holds for $r_{1}=1$. The induction step follows exactly the same proof and will be omitted.

\section{Proof of Theorem 3.2}

Following [Cou15] the proof of Theorem 3.2 is based on an energy-dissipation balance law which is obtained from the introduction of a multiplier inspired of the multiplier of the Leray-Gärding method [?]-[?] (see also Rau72 for an analogous approach in the continous framework). We will use exactly the same multiplier as in Cou15. Using the fact that this multiplier only depends on the discretization in the interior of the domain, we will show that this multiplier gives "strictly dissipative" boundaries and corner conditions and thus permits to introduce an auxiliary problem (posed in the full space, and as a consequence differing from the one used in Section (4) whose solution admits suitable (for the final error analysis) control of the traces to show the semi-group stability of (21).

Before we turn to a precise statement of the auxiliary problem, let us recall the definition of the multiplier used in Cou15] and the energy-dissipation balance law that it induces for finite difference schemes in the full space.

We define:

$$
L:=\sum_{\sigma=0}^{s+1} \mathbf{T}_{0}^{\sigma} Q^{\sigma}, \text { and } M:=\sum_{\sigma=0}^{s+1} \sigma \mathbf{T}_{0}^{\sigma} Q^{\sigma},
$$

where $\mathbf{T}_{0}^{\sigma}$ is the time-shifting operator.

Then we have the following balance law:

Lemma 5.1 (Cou15] Proposition 2) Under Assumptions 3.3 and 3.4 then there exist a continous coercive quadratic form $E$ and a continous nonnegative quadratic form $D$ on $\ell^{2}\left(\mathbb{Z}^{d}, \mathbb{R}\right)^{s+1}$ such that for all $\left(v^{n}\right)_{n \geq 0}$ with values in $\ell^{2}\left(\mathbb{Z}^{d}, \mathbb{R}\right)$ and all $n \in \mathbb{N}$ we have:

$$
2\left\langle\left\langle M v^{n}, L v^{n}\right\rangle\right\rangle_{\mathbb{Z}^{2}}=(s+1)\left\|L v^{n}\right\|_{\mathbb{Z}^{2}}^{2}+\left(\mathbf{T}_{0}-I\right) E\left(v^{n}, \ldots, v^{n+s}\right)+D\left(v^{n}, \ldots, v^{n+s}\right) .
$$


Note that we do not require that the sequence $\left(v_{j}^{n}\right)$ solves any finite difference scheme. Indeed Lemma 5.1 only depends on the coefficient of the discretization in the interior. This observation will be required to extend the proof of Cou15 to corner domains.

\subsection{Homogeneous initial conditions and auxiliary problem with strictly dissi- pative boundary and corner conditions}

As the proof of Theorem 3.1. the proof of Theorem 3.2 uses the linearity of 21 to treat separately the case of homogeneous initial conditions and the case of nonzero initial conditions. For homogeneous initial conditions the proof is a straightforward generalization of the proof in the half space. This proof is given in the following paragraph for a sake of completness. For nonzero initial conditions the proof needs to introduce an auxiliary problem which is posed in the full space and admits strictly dissipative boundary and corner conditions (compared with the proof in the half space where only one strictly dissipative boundary condition is needed).

\subsubsection{Proof of Theorem 3.2 for homogeneous initial conditions}

We first show Theorem 3.2 for homogeneous initial conditions that is:

Lemma 5.2 Under Assumptions 3.3 3.4 and 3.5, assume that the difference scheme approximation (21) is strongly stable in the sense of Definition 2.1 then $u$ the solution of 23 with homogeneous initial conditions satisfies that there exists $C>0$ such that for all $\gamma>0$ and $\Delta t \in] 0,1]$ we have the following estimate:

$$
\begin{aligned}
\sup _{n \geq 0} e^{-2 \gamma n \Delta t}\left\|u^{n}\right\|_{\mathscr{R}}^{2} & +\frac{\gamma}{\gamma \Delta t+1} \sum_{n \geq 0} \Delta t e^{-2 \gamma n \Delta t}\left\|u^{n}\right\|_{\mathscr{R}}^{2}+\sum_{k=1}^{2} \sum_{n \geq 0} \Delta t \Delta x_{3-k} e^{-2 \gamma n \Delta t}\left\|u^{n}\right\|_{\mathscr{B}_{k}}^{2} \leq \\
& C\left(\frac{\gamma \Delta t+1}{\gamma} \sum_{n \geq s+1} \Delta t e^{-2 \gamma n \Delta t}\left\|f^{n}\right\|_{\mathscr{I}}^{2}+\sum_{k=1}^{2} \sum_{n \geq s+1} \Delta t \Delta x_{3-k} e^{-2 \gamma n \Delta t}\left\|g_{k}^{n}\right\|_{\mathscr{B}_{k}}^{2}\right. \\
& \left.+\sum_{n \geq s+1} \Delta t \Delta x_{2} e^{-2 \gamma n \Delta t}\left\|h^{n}\right\|_{\mathscr{C}}^{2}\right) .
\end{aligned}
$$

Proof : By strong stability of (23) (recall that we assumed $u_{n} \equiv 0$ for $\left.n \in \llbracket 0, s \rrbracket\right)$ it is sufficient to show that $\sup _{n>0} e^{-2 \gamma n \Delta t}\left\|u^{n}\right\|_{\mathscr{R}}^{2}$ can be bounded by the right hand side of (46). As in the proof of Theorem 4.1 we introduce $\left(w_{j}^{n}\right)$ the extension of $\left(u_{j}^{n}\right)$ by zero for $j \in \mathbb{Z}^{2} \backslash \mathscr{R}$ (recall that we have $L w_{j}^{n}=0$ for $j_{1} \leq-\ell_{1}-r_{1}$ or $j_{2} \leq-\ell_{2}-r_{2}$ and $L w_{j}^{n}=L u_{j}^{n}$ for $j \in \mathscr{I}$, and so do for $M$ ). Applying Lemma 5.1. using the nonnegativity of $D$ it follows that:

$$
\left(\mathbf{T}_{0}-I\right) E\left(w^{n}, \ldots, w^{n+s}\right) \leq 2\left\langle\left\langle M w^{n}, L w^{n}\right\rangle\right\rangle_{\overline{\mathscr{R}} \backslash \mathscr{I}}-(s+1)\left\|L w^{n}\right\|_{\mathscr{\mathscr { R }} \backslash \mathscr{I}}^{2}+2 \Delta t\left\langle\left\langle M u^{n}, f^{n}\right\rangle\right\rangle_{\mathscr{I}}-(s+1) \Delta t^{2}\left\|f^{n}\right\|_{\mathscr{I}}^{2},
$$

where $\overline{\mathscr{R}}:=\mathscr{R} \cup \mathscr{E}_{\mathscr{B}_{1}} \cup \mathscr{E}_{\mathscr{B}_{2}} \cup \mathscr{E}_{\mathscr{C}}$ (see $(30)$ for the definition of the $\mathscr{E}_{\mathfrak{I}}$ ). Multiplying by $e^{-2 \gamma(n+s+1) \Delta t}$ and summing over $n \in \llbracket 0, N \rrbracket$ gives:

$$
e^{-2 \gamma(N+s+1) \Delta t} E\left(w^{N+1}, \ldots, w^{N+s+1}\right)+\left(1-e^{-2 \gamma \Delta t}\right) \sum_{n=1}^{N} e^{-2 \gamma(n+s) \Delta t} E\left(v^{n}, \ldots v^{n+s}\right) \leq I_{\overline{\mathscr{R}} \backslash \mathscr{I}, N}+I_{\mathscr{I}, N}
$$

with

$$
\begin{aligned}
I_{\overline{\mathscr{R}} \backslash \mathscr{I}, N} & :=\sum_{n=0}^{N} e^{-2 \gamma(n+s+1) \Delta t}\left(2\left\langle\left\langle M w^{n}, L w^{n}\right\rangle\right\rangle_{\overline{\mathscr{R}} \backslash \mathscr{I}}-(s+1)\left\|L w^{n}\right\|_{\mathscr{\mathscr { R }} \backslash \mathscr{I}}^{2}\right), \\
I_{\mathscr{I}, N} & :=\sum_{n=0}^{N} \Delta t e^{-2 \gamma(n+s+1) \Delta t}\left(2\left\langle\left\langle M u^{n}, f^{n}\right\rangle\right\rangle_{\mathscr{I}}-(s+1) \Delta t\left\|f^{n}\right\|_{\mathscr{I}}^{2}\right),
\end{aligned}
$$


and we will estimate these terms separately. First remark that by definition of $L, M$ and $\left(w_{j}^{n}\right)$ the terms $L w^{n}$ and $M w^{n}$ only involve the $u_{j}^{n+\sigma}$ for $j \in \overline{\mathscr{B}_{1}} \cup \overline{\mathscr{B}_{2}}$ and $\sigma \in \llbracket 0, s+1 \rrbracket$. By CFL condition we can always exchange in the equation defining $I_{\overline{\mathscr{R}} \backslash \mathscr{I}, N}$, the factor $\Delta x_{1}$ or $\Delta x_{2}$ by $\Delta t$ (see Section 4 for similar arguments) and we can thus use the trace estimate given by the strong stability of (21) and the trivial bound $I_{\overline{\mathscr{R}} \backslash \mathscr{I}, N} \leq I_{\overline{\mathscr{R}} \backslash \mathscr{I}, \infty}$ to show that $I_{\overline{\mathscr{R}} \backslash \mathscr{I}, N}$ is bounded by the right hand side of 46 .

We now turn to $I_{\mathscr{I}, N}$. For $j \in \mathscr{I}$ we remark that $M w_{j}^{n}$ only involves the $u_{j}^{n+\sigma}$ for $j \in \mathscr{R}$ and $\sigma \in \llbracket 0, s+1 \rrbracket$. As a consequence $\Delta t\left\|M w^{n}\right\|_{\mathscr{I}}$ only involves the $\Delta t\left\|u^{n+\sigma}\right\|_{\mathscr{R}}$ for $\sigma \in \llbracket 0, s+1 \rrbracket$. We use this time the interior estimate given by the strong stability assumption on (21) to bound these terms. Applying exactly the same computations as in Cou15. we show that $I_{\mathscr{I}, N}$ is also bounded by the right hand side of (46).

To conclude, we go back to the left hand side of (47) and from the coercivity of $E$ we have that:

$$
e^{-2 \gamma(N+s+1) \Delta t}|| v^{N+s+1} \mid \| \leq I_{\overline{\mathscr{R}} \backslash \mathscr{I}, N}+I_{\mathscr{I}, N},
$$

and 460 follows by taking the supremum in $N$.

Remark Note that compared to the proof of Theorem 3.1 it is here crucial (in view to obtain a suitable estimate for $I_{\overline{\mathscr{R}} \backslash \mathscr{I}, N}$ ) that the strong stability provides a control of the extended traces on $\overline{\mathscr{B}_{1}}$ and $\overline{\mathscr{B}_{2}}$ and not only a control of the traces on $\mathscr{B}_{1}$ and $\mathscr{B}_{2}$.

\subsubsection{Auxiliary problem}

In this paragraph we use the multiplier $M$ to introduce an auxiliary problem defined in the full space for which we can show a semi-group estimate and an extended traces estimate (which will be used in the end of the proof as it has been done in Section 4 to control the error terms).

More precisely the result is the following:

Theorem 5.1 ([Cou15], Theorem 2) Under Assumptions 3.3 3.4 and 3.5, for all $P_{1}, P_{2} \in \mathbb{N}$, there exists $C>0$ such that the solution $\left(u_{j}^{n}\right)$ of:

$$
\begin{cases}L u_{j}^{n}=0, & \text { for } n \geq 0, j \in \mathscr{I} \times \mathbb{Z}^{d-2}, \\ M u_{j}^{n}=g_{1, j}^{n+s+1}, & \text { for } n \geq 0, j_{1} \leq 0, j_{2} \geq 1, j^{\prime} \in \mathbb{Z}^{d-2}, \\ M u_{j}^{n}=g_{2, j}^{n+s+1}, & \text { for } n \geq 0, j_{1} \geq 1, j_{2} \leq 1, j^{\prime} \in \mathbb{Z}^{d-2}, \\ M u_{j}^{n}=h_{j}^{n+s+1}, & \text { for } n \geq 0, j_{1} \leq 0, j_{2} \leq 0, j^{\prime} \in \mathbb{Z}^{d-2} \\ u_{j}^{n}=u_{n, j}, & \text { for } n \in \llbracket 0, s \rrbracket, j \in \mathbb{Z}^{d},\end{cases}
$$

satisfies that for all $\gamma>0, \Delta t \in] 0,1]$ :

$$
\begin{aligned}
\sup _{n \geq 0} e^{-2 \gamma n \Delta t}\left\|u^{n}\right\|_{\mathbb{Z}^{2}}^{2} & +\frac{\gamma}{\gamma \Delta t+1} \sum_{n \geq 0} \Delta t e^{-2 \gamma n \Delta t}\left\|u^{n}\right\|_{\mathbb{Z}^{2}}^{2}+\sum_{k=1}^{2} \sum_{n \geq 0} \Delta t \Delta x_{3-k} e^{-2 \gamma n \Delta t} \sum_{j_{k}=1-\ell_{k}}^{P_{k}}\left\|u_{j_{k}}^{n},\right\|_{\ell^{2}\left(\mathbb{Z}^{d-1}\right)}^{2} \\
& \leq C\left(\sum_{n=0}^{s}\left\|u_{n}\right\|_{\mathbb{Z}^{2}}^{2}+\sum_{k=1}^{2} \sum_{n \geq s+1} \Delta t \Delta x_{3-k} e^{-2 \gamma n \Delta t} \sum_{j_{k} \leq 0, j_{3-k} \geq 1}\left\|g_{k, j_{k}, j_{3-k}}^{n} \cdot\right\|_{\ell^{2}\left(\mathbb{Z}^{d-2}\right)}^{2}\right. \\
& \left.+\sum_{n \geq s+1} \Delta t \Delta x_{2} e^{-2 \gamma n \Delta t} \sum_{j_{1} \leq 0, j_{2} \leq 0}\left\|h_{j_{k}, j_{3-k},}^{n},\right\|_{\ell^{2}\left(\mathbb{Z}^{d-2}\right)}^{2}\right)
\end{aligned}
$$

Before we turn to the proof of Theorem 5.1 let us give some arguments explaining why essentially the same as the proof of Theorem 2 in Cou15 will effectively operate even if the auxiliary problems differ. Indeed compared to the auxiliary problem introduced in Cou15, the auxiliary problem (48) is defined by $M u_{j}^{n}=g_{2, j}^{n+s+1}$ and not $L u_{j}^{n}=0$ in the quarter space $j_{1} \geq 1, j_{2} \leq 1, j^{\prime} \in \mathbb{Z}^{d-2}$. Moreover the proof of 49] given in [Cou15] relies on partial Fourier transform which are not a priori suitable in the geometry of the 
quarter space due to the fact that "there are too many normal directions".

However, using the fact that $\left(u_{j}^{n}\right)$ the solution of 48 is defined in the full space $\mathbb{Z}^{d}$ any extension will be needed to perform partial Fourier transform and consequently, in this particular setting, we will be able to perform two partial Fourier transforms to obtain the estimate 49 (one transform by trace that have to be controled). The fact that $\left(u_{j}^{n}\right)$ does not solve $L u_{j}^{n}=0$ in the quarter space $j_{1} \geq 1, j_{2} \leq 1, j^{\prime} \in \mathbb{Z}^{d-2}$ will not be an issue neither. Indeed, in Cou15] the main part of the proof leading to (49) does not strongly use the equation solved by $\left(u_{j}^{n}\right)$. Indeed here are the main points in the proof of [Cou15].

Firstly one uses Lemma 5.1 to obtain an estimate of $\sup _{n \geq 0} e^{-2 \gamma n \Delta t}\left\|u^{n}\right\|_{\mathbb{Z}^{2}}^{2}, \sum_{n \geq 0} \Delta t e^{-2 \gamma n \Delta t}\left\|u^{n}\right\| \|_{\mathbb{Z}^{2}}^{2}$ and $\sum_{n \geq 0} e^{-2 \gamma(n+s+1) \Delta t}\left\|L u^{n}\right\|_{\mathbb{Z}^{2}}^{2}$.

Then to remplace $\sum_{n \geq 0} e^{-2 \gamma(n+s+1) \Delta t}\left\|L u^{n}\right\|_{\mathbb{Z}^{2}}^{2}$ by $\sum_{k=1}^{2} \sum_{n \geq 0} \Delta t \Delta x_{3-k} e^{-2 \gamma n \Delta t} \sum_{j_{k}=1-\ell_{k}}^{P_{k}}\left\|u_{j_{k}}^{n},\right\|_{\ell^{2}\left(\mathbb{Z}^{d-1}\right)}^{2}$ in this estimate, the arguments are the following. In a first time one works in the regime $\gamma \Delta t$ large and only needs algebraic properties of $L$ (but does not use the fact that $\left(u_{j}^{n}\right)$ solves a precise difference scheme) and thus in this regime we will be able to apply the same proof as in Cou15.

Then, in the regime $\gamma \Delta t$ small, the first step is to use the estimate to show that Laplace-Fourier transform of $\left(u_{j}^{n}\right)$ is well-defined. Then by algebraic computations and using the fact that $\left(u_{j}^{n}\right)$ solves the boundary conditions (but not the fact that it solves $L u_{j}^{n}=0$ on any quarter space) one obtains that some algebraic quantity involving the Laplace-Fourier transform of $\left(u_{j}^{n}\right)$ and the symbol of $L$ is bounded by the right hand side of 49). To conclude its remains to show that the considered algebraic quantity controls the extended traces for any considered sequence. This last point is independent of the defintion of $\left(u_{j}^{n}\right)$ and so it will also works if $\left(u_{j}^{n}\right)$ solves 48 .

Proof : Firstly we apply Lemma 5.1 to 48, in view to demonstrate the analogous estimate than in Cou15. From the nonnegativity of D it follows that:

$$
\begin{aligned}
\left(\mathbf{T}_{0}-I\right) E\left(u^{n}, \ldots, u^{n+s}\right)+(s+1)\|\| L u^{n} \|_{\mathbb{Z}^{2}}^{2} & \leq 2\left\langle\left\langle g_{1}^{n}, L u^{n}\right\rangle\right\rangle_{\rrbracket-\infty, 0 \rrbracket \times \llbracket 1, \infty \llbracket}+2\left\langle\left\langle g_{2}^{n}, L u^{n}\right\rangle\right\rangle_{\llbracket 1, \infty \llbracket \times \rrbracket-\infty, 0 \rrbracket} \\
& +2\left\langle\left\langle h^{n}, L u^{n}\right\rangle\right\rangle_{\rrbracket-\infty, 0 \rrbracket^{2}},
\end{aligned}
$$

we then use three times Cauchy-Schwartz combined with Young inequality (with parameter $\frac{(s+1)}{6}$ ) to obtain:

$$
\left(\mathbf{T}_{0}-I\right) E\left(u^{n}, \ldots, u^{n+s}\right)+\frac{(s+1)}{2}\left\|L u^{n}\right\|_{\mathbb{Z}^{2}}^{2} \leq 6\left(\left\|g_{1}^{n}\right\|_{\rrbracket-\infty, 0 \rrbracket \times \llbracket 1, \infty \llbracket}^{2}+\left\|g_{2}^{n}\right\|_{\llbracket 1, \infty \llbracket \times \rrbracket-\infty, 0 \rrbracket}^{2}+\left\|h^{n}\right\|_{\rrbracket-\infty, 0 \rrbracket^{2}}^{2}\right) .
$$

We then multiply the latter inequality by $e^{-2 \gamma(n+s+1) \Delta t}$ and sum from $n=0$ to $N$. Reiterating the same kind of computations as in Section 4 we obtain, from the coercivity of $E$ the following estimate:

$$
\begin{aligned}
& \sup _{n \geq 0} e^{-2 \gamma n \Delta t}\left\|u^{n}\right\|_{\mathbb{Z}^{2}}^{2}+\frac{\gamma}{\gamma \Delta t+1} \sum_{n \geq 0} \Delta t e^{-2 \gamma n \Delta t}\left\|u^{n}\right\|_{\mathbb{Z}^{2}}^{2}+\sum_{n \geq 0} e^{-2 \gamma(n+s+1) \Delta t}\left\|L u^{n}\right\|_{\mathbb{Z}^{2}}^{2} \leq \\
& C \quad\left(\sum_{n=0}^{s}\left\|u_{n}\right\|_{\mathbb{Z}^{2}}^{2}+\sum_{k=1}^{2} \sum_{n \geq s+1} \Delta t \Delta x_{3-k} e^{-2 \gamma n \Delta t} \sum_{j_{k} \leq 0, j_{3-k} \geq 1}\left\|g_{k, j_{k}, j_{3-k} .}^{n}\right\|_{\ell^{2}\left(\mathbb{Z}^{d-2}\right)}^{2}\right. \\
& \left.+\sum_{n \geq s+1} \Delta t \Delta x_{2} e^{-2 \gamma n \Delta t} \sum_{j_{1} \leq 0, j_{2} \leq 0}\left\|h_{j_{k}, j_{3-k}}^{n},\right\|_{\ell^{2}\left(\mathbb{Z}^{d-2}\right)}^{2}\right) \text {. }
\end{aligned}
$$

Using the definition of the CFL numbers we can rewrite the weight $\Delta x_{1} \Delta x_{2}$ as $\Delta t \Delta x_{2}$ or $\Delta t \Delta x_{1}$. So $(50)$ 
can also be rewritten under the form:

$$
\begin{aligned}
\sup _{n \geq 0} e^{-2 \gamma n \Delta t}\left\|u^{n}\right\|_{\mathbb{Z}^{2}}^{2} & +\frac{\gamma}{\gamma \Delta t+1} \sum_{n \geq 0} \Delta t e^{-2 \gamma n \Delta t}\left\|u^{n}\right\|_{\mathbb{Z}^{2}}^{2}+\sum_{k=1}^{2} \sum_{n \geq 0} \Delta t \Delta x_{3-k} \sum_{j_{k} \in \mathbb{Z}} e^{-2 \gamma(n+s+1) \Delta t}\left\|L u_{j_{k}}^{n},\right\|_{\ell^{2}\left(\mathbb{Z}^{d-1}\right)}^{2} \leq \\
& C\left(\sum_{n=0}^{s}\left\|u_{n}\right\|_{\mathbb{Z}^{2}}^{2}+\sum_{k=1}^{2} \sum_{n \geq s+1} \Delta t \Delta x_{3-k} e^{-2 \gamma n \Delta t} \sum_{j_{k} \leq 0, j_{3-k} \geq 1}\left\|g_{k, j_{k}, j_{3-k}}^{n} .\right\|_{\ell^{2}\left(\mathbb{Z}^{d-2}\right)}^{2}\right. \\
& \left.+\sum_{n \geq s+1} \Delta t \Delta x_{2} e^{-2 \gamma n \Delta t} \sum_{j_{1} \leq 0, j_{2} \leq 0}\left\|h_{j_{k}, j_{3-k},}^{n},\right\|_{\ell^{2}\left(\mathbb{Z}^{d-2}\right)}^{2}\right) .
\end{aligned}
$$

To conclude the proof of Theorem 5.1 it is sufficient to explain how (51) implies a good control of the extended traces values $\sum_{k=1}^{2} \sum_{n \geq 0} \Delta t \Delta x_{3-k} e^{-2 \gamma n \Delta t} \sum_{j_{k}=1-\ell_{k}}^{P_{k}}\left\|u_{j_{k}}^{n},\right\|_{\ell^{2}\left(\mathbb{Z}^{d-1}\right)}^{2}$.

Following Cou15 in view to do this it is sufficient to distinguish two cases depending on the value of $\gamma \Delta t$. The proof in the framework $\gamma \Delta t$ large is totally analogous (because as already mentioned it only uses algebraic properties of the operator $L$ ) to the one given in Cou15 and will not be repeated here. Let us recall that when $\gamma \Delta t$ is large we, in fact, obtain the control of infinitely many traces:

$$
\begin{aligned}
\sum_{k=1}^{2} \sum_{n \geq 0} \Delta t \Delta x_{3-k} e^{-2 \gamma n \Delta t} \sum_{j_{k} \in \mathbb{Z}}\left\|u_{j_{k},}^{n},\right\|_{\ell^{2}\left(\mathbb{Z}^{d-1}\right)}^{2} \leq & \\
C\left(\sum_{n=0}^{s}\left\|u_{n}\right\|_{\mathbb{Z}^{2}}^{2}+\sum_{k=1}^{2} \sum_{n \geq s+1} \Delta t \Delta x_{3-k} e^{-2 \gamma n \Delta t} \sum_{j_{k} \leq 0, j_{3-k} \geq 1}\left\|g_{k, j_{k}, j_{3-k}}^{n}\right\|_{\ell^{2}\left(\mathbb{Z}^{d-2}\right)}^{2}\right. & \left.+\sum_{n \geq s+1} \Delta t \Delta x_{2} e^{-2 \gamma n \Delta t} \sum_{j_{1} \leq 0, j_{2} \leq 0}\left\|h_{j_{k}, j_{3-k},}^{n},\right\|_{\ell^{2}\left(\mathbb{Z}^{d-2}\right)}^{2}\right),
\end{aligned}
$$

and not only a control of finitely many traces.

We now turn to the case $\gamma \Delta t$ small. As the reader will see the proof in this framework will not need any adaptation of the proof given in Cou15. However as the proof of Cou15] relies on partial Fourier transform (which was the only point that we needed to adapt in the proof of Theorem 3.1) it seems important, in the author opinion, to give some comments about the previous claim.

Firstly the estimate (51) shows that the Laplace in time and partial Fourier transforms in spaces $\left(j_{1}, j^{\prime}\right)$ and $\left(j_{2}, j^{\prime}\right)$ of $\left(u_{j}^{n}\right)$ are well-defined. We denote these transforms by ${\widehat{u_{j_{2}}}}^{1}$ and ${\widehat{u_{j_{1}}}}^{2}$ respectively. We introduce $\tau:=\gamma+i \theta$ the dual variable of time for the Laplace transform and $\eta_{k}:=\left(j_{k}, j^{\prime}\right)$ the dual variable of space for the partial Fourier transform in space $\left(j_{k}, j^{\prime}\right)$. To save some notations we also denote $\eta_{k, \Delta}:=$ $\left(j_{k}, j^{\prime}\right) \cdot\left(\Delta x_{k}, \Delta x^{\prime}\right)$.

The following lemma gives a control of ${\widehat{u_{j_{2}}}}^{1}$ and ${\widehat{u_{j_{1}}}}^{2}$ up to some multiplication by the associated symbols of $L$ and $M$ in term of the right hand side of 49 if $\gamma \Delta t<\ln R_{0}$ for some fixed $R_{0}>1$ :

Lemma 5.3 There exists $C>0$ such that for all $\gamma>0, \Delta t \in] 0,1]$ satisfying $\gamma \Delta t<\ln R_{0}$, we have that for 
$k \in \llbracket 1,2 \rrbracket:$

$$
\begin{aligned}
\sum_{j_{k} \in \mathbb{Z}} \int_{\mathbb{R} \times \mathbb{R}^{d-1}} & \left|\sum_{\mu_{k}=-\ell_{k}}^{r_{k}} a^{\mu_{k}}\left(e^{\tau \Delta t}, \eta_{\Delta, 3-k}\right){\widehat{u_{j}}}^{3-k}\left(\tau, \eta_{3-k}\right)\right|^{2} d \theta d \eta_{3-k} \\
& +\sum_{j_{k} \leq 0} \int_{\mathbb{R} \times \mathbb{R}^{d-1}}\left|\sum_{\mu_{k}=-\ell_{k}}^{r_{k}} e^{\tau \Delta t} \partial_{z} a^{\mu_{k}}\left(e^{\tau \Delta t}, \eta_{\Delta, 3-k}\right){\widehat{u_{j}}}^{3-k}\left(\tau, \eta_{3-k}\right)\right|^{2} d \theta d \eta_{3-k} \\
\leq & C\left(\sum_{n=0}^{s}\left\|u_{n}\right\|_{\mathbb{Z}^{2}}^{2}+\sum_{k=1}^{2} \sum_{n \geq s+1} \Delta t \Delta x_{3-k} e^{-2 \gamma n \Delta t} \sum_{j_{k} \leq 0, j_{3-k} \geq 1}\left\|g_{k, j_{k}, j_{3-k}}^{n} \cdot\right\|_{\ell^{2}\left(\mathbb{Z}^{d-2}\right)}+\sum_{n \geq s+1} \Delta t \Delta x_{2} e^{-2 \gamma n \Delta t} \sum_{j_{1} \leq 0, j_{2} \leq 0}\left\|h_{j_{k}, j_{3-k},}^{n},\right\|_{\ell^{2}\left(\mathbb{Z}^{d-2}\right)}^{2}\right) .
\end{aligned}
$$

Then the following lemma gives the control of the trace of any sequence in term of the left hand side of the inequality appearing in Lemma 5.3 . More precisely, we have:

Lemma 5.4 Under Assumptions 3.3 3.4 and 3.5, for $k \in \llbracket 1,2 \rrbracket$ and for any fixed $P_{k} \in \mathbb{N}$, there exists $C_{P_{k}}>0$ such that for all $z \in \mathbb{C}$ such that $1 \leq|z| \leq R_{0}$, for all $\eta_{3-k} \in \mathbb{R}^{d-1}$ and for all sequence $\left(w_{j_{k}}\right)_{j_{k} \in \mathbb{Z}} \in \ell^{2}(\mathbb{Z}, \mathbb{C})$ we have:

$$
\begin{aligned}
\sum_{j_{k}=-\ell_{k}-r_{k}}^{P_{k}}\left|w_{j_{k}}\right|^{2} \leq C_{P_{k}} & \left(\sum_{j_{k} \in \mathbb{Z}} \int_{\mathbb{R} \times \mathbb{R}^{d-1}}\left|\sum_{\mu_{k}=-\ell_{k}}^{r_{k}} a^{\mu_{k}}\left(e^{\tau \Delta t}, \eta_{\Delta, 3-k}\right) w_{j_{k}+\mu_{k}}\right|^{2} d \theta d \eta_{3-k}\right. \\
& \left.+\sum_{j_{k} \leq 0} \int_{\mathbb{R} \times \mathbb{R}^{d-1}}\left|\sum_{\mu_{k}=-\ell_{k}}^{r_{k}} e^{\tau \Delta t} \partial_{z} a^{\mu_{k}}\left(e^{\tau \Delta t}, \eta_{\Delta, 3-k}\right) w_{j_{k}+\mu_{k}}\right|^{2} d \theta d \eta_{3-k}\right) .
\end{aligned}
$$

With Lemmas 5.3 and 5.4 in hand let us describe how to conclude the proof of Theorem 5.1 For $k \in \llbracket 1,2 \rrbracket$,

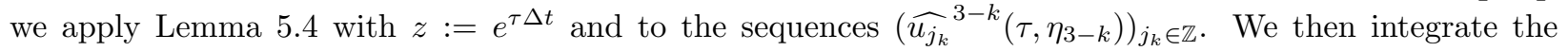
estimate of Lemma 5.4 with respect to $\left(\theta, \eta_{3-k}\right)$ and choose the real part of $\tau$ small enougt to apply Lemma 5.3. We thus have:

$$
\begin{array}{r}
\sum_{j_{k}=-\ell_{k}-r_{k}}^{P_{k}} \int_{\mathbb{R} \times \mathbb{R}^{d-1}}\left|w_{j_{k}}\right|^{2} d \theta d \eta_{3-k} \leq C\left(\sum_{n=0}^{s}\left\|u_{n}\right\|_{\mathbb{Z}^{2}}^{2}+\sum_{k=1}^{2} \sum_{n \geq s+1} \Delta t \Delta x_{3-k} e^{-2 \gamma n \Delta t} \sum_{j_{k} \leq 0, j_{3-k} \geq 1}\left\|g_{k, j_{k}, j_{3-k},}^{n} \cdot\right\|_{\ell^{2}\left(\mathbb{Z}^{d-2}\right)}^{2}\right) \\
\left.+\sum_{n \geq s+1} \Delta t \Delta x_{2} e^{-2 \gamma n \Delta t} \sum_{j_{1} \leq 0, j_{2} \leq 0}\left\|h_{j_{k}, j_{3-k},}^{n},\right\|_{\ell^{2}\left(\mathbb{Z}^{d-2}\right)}^{2}\right) .
\end{array}
$$

To come back in terms of the sequence $\left(u_{j_{k}}^{n}\right.$, ) we apply Plancherel formula in the left hand side and use the fact that $\gamma \Delta t$ is small to obtain that:

$$
\begin{aligned}
\sum_{j_{k}=-\ell_{k}-r_{k}}^{P_{k}} \sum_{n \geq 0} \Delta t e^{-2 \gamma n \Delta t}\left\|u_{j_{k}},\right\|_{\ell^{2}\left(\mathbb{Z}^{d-1}\right)}^{2} & \leq \sum_{j_{k}=-\ell_{k}-r_{k}}^{P_{k}} \sum_{n \geq 0} \frac{1-e^{-2 \gamma \Delta t}}{2 \gamma \Delta t} \Delta t e^{-2 \gamma n \Delta t}\left\|u_{j_{k}},\right\|_{\ell^{2}\left(\mathbb{Z}^{d-1}\right)}^{2} \\
& =\sum_{j_{k}=-\ell_{k}-r_{k}}^{P_{k}} \int_{\mathbb{R} \times \mathbb{R}^{d-1}}\left|w_{j_{k}}\right|^{2} d \theta d \eta_{3-k},
\end{aligned}
$$

which concludes the proof of Theorem 5.1 . 
Remark We conclude this paragraph by giving some elements of proof for Lemmas 5.3 and 5.4 . The proof of 5.4 is the most technical one but since it only depends on the tangential operators $a^{\mu_{k}}$, we will not reiterate it here. The proof of Lemma 5.3 uses the fact that the considered sequence solves an explicit scheme. However this point is only used to establish the estimate (51). As we have already seen this estimate also holds for our choice of auxiliary scheme and consequently we can reiterate exactly the proof given in Cou15 to show Lemma 5.3.

\subsection{End of the proof by error estimate}

The case of finite difference schemes with homogeneous initial conditions has already been treated in Paragraph 5.1. So without loss of generality we can assume that in 23 the sequences $\left(f_{j}^{n}\right),\left(g_{1, j}^{n}\right),\left(g_{2, j}^{n}\right)$ and $\left(h_{j}^{n}\right)$ are zero. We denote the associated solution by $\left(u_{j}^{n}\right)$. By linearity of 23 we decompose $\left(u_{j}^{n}\right)$ into $u_{j}^{n}:=v_{j}^{n}+w_{j}^{n}$ where $\left(v_{j}^{n}\right)$ is the solution of the auxiliary problem with strictly dissipative boundary and corner conditions:

$$
\begin{cases}L v_{j}^{n}=0, & \text { for } n \geq 0, j \in \mathscr{I} \times \mathbb{Z}^{d-2}, \\ M v_{j}^{n}=0, & \text { for } n \geq 0, j_{1} \leq 0, j_{2} \geq 1, j^{\prime} \in \mathbb{Z}^{d-2}, \\ M v_{j}^{n}=0, & \text { for } n \geq 0, j_{1} \geq 1, j_{2} \leq 1, j^{\prime} \in \mathbb{Z}^{d-2}, \\ M v_{j}^{n}=0, & \text { for } n \geq 0, j_{1} \leq 0, j_{2} \leq 0, j^{\prime} \in \mathbb{Z}^{d-2}, \\ v_{j}^{n}=\widetilde{u}_{n, j}, & \text { for } n \in \llbracket 0, s \rrbracket, j \in \mathbb{Z}^{d},\end{cases}
$$

where $\left(\widetilde{u}_{n, j}\right)$ is the extension of $\left(u_{n, j}\right)$ by zero for $j \neq \mathscr{R}$; and where $\left(w_{j}^{n}\right)$ is the solution of 23] with homogeneous initial conditions (but inhomogeneous boundary and corner conditions):

$$
\begin{cases}L w_{j}^{n}=0, & \text { for } n \geq 0, j \in \mathscr{I} \times \mathbb{Z}^{d-2}, \\ w_{j}^{n+s+1}+\sum_{\sigma=0}^{s+1} B_{1}^{\sigma, j_{1}} w_{j}^{n+\sigma}=\widetilde{g}_{1, j}^{n+s+1}, & \text { for } j \in \mathscr{B}_{1} \times \mathbb{Z}^{d-2}, n \geq 0, \\ w_{j}^{n+s+1}+\sum_{\sigma=0}^{s+1} B_{2}^{\sigma, j_{2}} w_{j}^{n+\sigma}=\widetilde{g}_{2, j}^{n+s+1}, & \text { for } j \in \mathscr{B}_{2} \times \mathbb{Z}^{d-2}, n \geq 0, \\ w_{j}^{n+s+1}+\sum_{\sigma=0}^{s+1} C^{\sigma, j_{1}, j_{2}} w_{j}^{n+\sigma}=\widehat{h}_{j}^{n+s+1}, & \text { for } j \in \mathscr{C} \times \mathbb{Z}^{d-2}, n \geq 0, \\ w_{j}^{n}=0, & \text { for } j \in \mathscr{R} \times \mathbb{Z}^{d-2}, n \in \llbracket 0, s \rrbracket .\end{cases}
$$

where $\widetilde{g}_{k, j}^{n+s+1}$ and $\widetilde{h}_{j}^{n+s+1}$ are the errors at the boundaries and at the corner induced by the sequence $\left(v_{j}^{n}\right)$. More precisely they are defined by:

$$
\begin{aligned}
\text { for } k \in \llbracket 1,2 \rrbracket, \quad \widetilde{g}_{k, j}^{n+s+1}:=-v_{j}^{n+s+1}-\sum_{\sigma=0}^{s+1} B_{k}^{\sigma, j_{k}} v_{j}^{n+\sigma}, \text { for } j \in \mathscr{B}_{k} \times \mathbb{Z}^{d-2}, \\
\text { and } \widetilde{h}_{j}^{n+s+1}:=-v_{j}^{n+s+1}-\sum_{\sigma=0}^{s+1} C^{\sigma, j_{1}, j_{2}} v_{j}^{n+\sigma}, \text { for } j \in \mathscr{C} \times \mathbb{Z}^{d-2} .
\end{aligned}
$$

Using the fact that (53) admits homogeneous initial conditions we can apply the estimate obtained in Paragraph 5.1.1. We thus have:

$$
\begin{aligned}
\sup _{n \geq 0} e^{-2 \gamma n \Delta t}\left\|w^{n}\right\|_{\mathscr{R}}^{2} & +\frac{\gamma}{\gamma \Delta t+1} \sum_{n \geq 0} \Delta t e^{-2 \gamma n \Delta t}\left\|w^{n}\right\|_{\mathscr{R}}^{2}+\sum_{k=1}^{2} \sum_{n \geq 0} \Delta t \Delta x_{3-k} e^{-2 \gamma n \Delta t}\left\|w^{n}\right\|_{\mathscr{B}_{k}}^{2} \leq \\
C & \left(\sum_{k=1}^{2} \sum_{n \geq s+1} \Delta t \Delta x_{3-k} e^{-2 \gamma n \Delta t}\left\|\widetilde{g}_{k}^{n}\right\|_{\mathscr{B}_{k}}^{2}+\sum_{n \geq s+1} \Delta t \Delta x_{2} e^{-2 \gamma n \Delta t}\left\|\widetilde{h}^{n}\right\|_{\mathscr{C}}^{2}\right)
\end{aligned}
$$

and we want to estimate the errors terms $\widetilde{g}_{k, j}^{n+s+1}$ and $\widetilde{h}_{j}^{n+s+1}$ in terms of the initial datas. In view of (54) from the triangle inequality we deduce that to conclude it is sufficient to control the norms of the terms $-v_{j}^{n+s+1}+\sum_{\sigma=0}^{s+1} B_{k}^{\sigma, j_{k}} v_{j}^{n+\sigma}$ and $-v_{j}^{n+s+1}+\sum_{\sigma=0}^{s+1} C^{\sigma, j_{1}, j_{2}} v_{j}^{n+\sigma}$ by the initial datas. To do this we used the strengthened traces and corner estimates obtained for $\left(v_{j}^{n}\right)$ in Theorem 5.1

As it has been done in the proof of Theorem 3.1 (see Paragraph 4.3) a discussion depending on the kind of the boundaries and corner conditions in 10 is needed. However as the arguments are totally similar to 
these described in the proof of Theorem 3.1 we will only here describe the proof when 10 admits decoupled traces and corner conditions (the proofs in the others cases are the same up to different values of $P_{1}$ and $P_{2}$ (see again Paragraph 4.3p).

Note that in view of the definition of $B_{1}^{\sigma, j_{1}}$ when 10 admits decoupled traces and corner conditions, for fixed $n$ and $j \in \mathscr{B}_{1} \times \mathbb{Z}^{d-2}$, the term $-v_{j}^{n+s+1}+\sum_{\sigma=0}^{s+1} B_{1}^{\sigma, j_{1}} v_{j}^{n+\sigma}$ only involve the $v_{\tilde{j}}^{n+\sigma}$ for $\tilde{j} \in \llbracket 1-$ $\ell_{1}, q_{11} \rrbracket \times \llbracket 1, \infty \llbracket \times \mathbb{Z}^{d-2}$ and $\sigma \in \llbracket 0, s+1 \rrbracket$. The triangle inequality and the estimate (49) applied to $P_{1}:=$ $\max \left\{r_{1}, 1+q_{11}\right\}$ then give (recall that 52 is homogeneous at the boundary):

$$
\begin{aligned}
\sum_{n \geq s+1} \Delta t \Delta x_{2} e^{-2 \gamma n \Delta t}\left\|\widetilde{g}_{1}^{n}\right\|_{\mathscr{B}_{1}}^{2} & \leq C \sum_{n \geq 0} \Delta t \Delta x_{2} e^{-2 \gamma n \Delta t} \sum_{j_{1}=1-\ell_{1}}^{P_{1}}\left\|v_{j_{1},}^{n}\right\|_{\ell^{2}\left(\mathbb{Z}^{d-1}\right)}^{2} \\
& \leq C \sum_{n=0}^{s}\left\|\widetilde{u}_{n, \cdot}\right\|_{\mathbb{Z}^{2}}^{2} .
\end{aligned}
$$

We then apply exactly the same arguments to estimate $\widetilde{g}_{2, j}^{n+s+1}$ (choosing $P_{2}=\max \left\{r_{2}, 1+q_{22}\right\}$ in 490 ) and we to obtain that:

$$
\sum_{n \geq s+1} \Delta t \Delta x_{1} e^{-2 \gamma n \Delta t}\left\|\widetilde{g}_{2}^{n}\right\|_{\mathscr{B}_{2}}^{2} \leq C \sum_{n=0}^{s}\left\|\widetilde{u}_{n,} \cdot\right\|_{\mathbb{Z}^{2}}^{2} .
$$

Finally to deal with $\widetilde{h}_{j}^{n+s+1}$ we reiterate a last time the previous reasoning with $P_{1}=\max \left\{r_{1}, 1+c_{11}\right\}$ in the estimate 49 to obtain:

$$
\sum_{n \geq s+1} \Delta t \Delta x_{2} e^{-2 \gamma n \Delta t}\left\|\widetilde{h}^{n}\right\|_{\mathscr{C}}^{2} \leq C \sum_{n=0}^{s}\left\|\widetilde{u}_{n,} \cdot\right\|_{\mathbb{Z}^{2}}^{2} .
$$

As a consequence we have that

$$
\begin{aligned}
\sup _{n \geq 0} e^{-2 \gamma n \Delta t}\left\|w^{n}\right\|_{\mathscr{R}}^{2}+\frac{\gamma}{\gamma \Delta t+1} \sum_{n \geq 0} \Delta t e^{-2 \gamma n \Delta t}\left\|w^{n}\right\|_{\mathscr{R}}^{2}+\sum_{k=1}^{2} \sum_{n \geq 0} \Delta t \Delta x_{3-k} e^{-2 \gamma n \Delta t}\left\|w^{n}\right\|_{\mathscr{B}_{k}}^{2} \leq \\
C \sum_{n=0}^{s}\left\|\widetilde{u}_{n, \cdot}\right\|_{\mathbb{Z}^{2}}^{2},
\end{aligned}
$$

and the same estimate holds for $\left(v_{j}^{n}\right.$ ) (by 49) for $P_{1}=r_{1}$ and $P_{2}=r_{2}$ ). We thus obtain 24 by the triangle inequality and from the fact that, in view of its definition, $\left\|\widetilde{u}_{n,} \cdot\right\|_{\mathbb{Z}^{2}}^{2}=\left\|\widetilde{u}_{n, \cdot}\right\|_{\mathscr{R}}^{2}$.

\section{References}

[Ben15] A. Benoit. Problèmes aux limites, optique géométrique et singularités. PhD thesis, Université de Nantes, 2015. https://hal.archives-ouvertes.fr/tel-01180449v1

[Ber94] Jean-Pierre Berenger. A perfectly matched layer for the absorption of electromagnetic waves. J. Comput. Phys., 114(2):185-200, 1994.

[BG07] S. Benzoni-Gavage, D. Serre. Multidimensional hyperbolic partial differential equations. Oxford Mathematical Monographs. Oxford University Press, 2007.

[BGS72] H.-O. Kreiss B. Gustafsson and A. Sundstrom. Stability theory of difference approximations for mixed initial boundary value problems. ii. Math. Comp.,, 26(119):649-686, 1972.

[CG11] Jean-François Coulombel and Antoine Gloria. Semigroup stability of finite difference schemes for multidimensional hyperbolic initial-boundary value problems. Math. Comp., 80(273):165-203, 2011. 
[Cou09] Jean-François Coulombel. Stability of finite difference schemes for hyperbolic initial boundary value problems. SIAM J. Numer. Anal., 47(4):2844-2871, 2009.

[Cou11] Jean-François Coulombel. Stability of finite difference schemes for hyperbolic initial boundary value problems II. Ann. Sc. Norm. Super. Pisa Cl. Sci. (5), 10(1):37-98, 2011.

[Cou15] Jean-François Coulombel. The Leray-Gårding method for finite difference schemes. J. Éc. polytech. Math., $2: 297-331,2015$.

[CP81] Jacques Chazarain and Alain Piriou. Introduction à la théorie des équations aux dérivées partielles linéaires. Gauthier-Villars, Paris, 1981.

[Ehr10] Matthias Ehrhardt. Absorbing boundary conditions for hyperbolic systems. Numer. Math. Theory Methods Appl., 3(3):295-337, 2010.

[EM77] Björn Engquist and Andrew Majda. Absorbing boundary conditions for numerical simulation of waves. Proc. Nat. Acad. Sci. U.S.A., 74(5):1765-1766, 1977.

[Gär56] Lars Gärding. Solution directe du problème de Cauchy pour les équations hyperboliques. In La théorie des équations aux dérivées partielles. Nancy, 9-15 avril 1956, Colloques Internationaux du Centre National de la Recherche Scientifique, LXXI, pages 71-90. Centre National de la Recherche Scientifique, Paris, 1956.

[Hig86] Robert L. Higdon. Absorbing boundary conditions for difference approximations to the multidimensional wave equation. Math. Comp., 47(176):437-459, 1986.

[HR] L. Halpern and J. Rauch. Well posedness of perfectly matched or dissipative boundary conditions with trihedral corners (to appear).

[Kre70] H.-O. Kreiss. Initial boundary value problems for hyperbolic systems. Comm. Pure Appl. Math., 23:277-298, 1970.

[Ler53] Jean Leray. Hyperbolic differential equations. The Institute for Advanced Study, Princeton, N. J., 1953.

[Osh73] S. Osher. Initial-boundary value problems for hyperbolic systems in regions with corners. I. Trans. Amer. Math. Soc., 176:141-164, 1973.

[Rau72] Jeffrey Rauch. $\mathcal{L}_{2}$ is a continuable initial condition for Kreiss' mixed problems. Comm. Pure Appl. Math., 25:265-285, 1972.

[Wu95] Lixin $\mathrm{Wu}$. The semigroup stability of the difference approximations for initial-boundary value problems. Math. Comp., 64(209):71-88, 1995. 